\title{
Symmetry-based design of fragment separator optics
}

\author{
B. Erdelyi* \\ Department of Physics, Northern Illinois University, DeKalb, Illinois 60115, USA \\ Physics Division, Argonne National Laboratory, Argonne, Illinois 60439, USA \\ J. Maloney \\ Department of Physics, Northern Illinois University, DeKalb, Illinois 60115, USA \\ J. Nolen \\ Physics Division, Argonne National Laboratory, Argonne, Illinois 60439, USA \\ (Received 16 February 2007; published 13 June 2007)
}

\begin{abstract}
Next-generation high-intensity large acceptance fragment separators require a careful design due to the large high order aberrations induced by the large aperture superconducting magnets needed to collect rare isotopes obtained from a high energy primary heavy-ion beam hitting a target. In this paper we propose a fragment separator layout based on various symmetries that satisfies the baseline requirements. Analytical calculations based on symmetry theories simplify the design to numerical optimization of a basic cell with only a few magnetic elements. The insight provided by these calculations resulted in the specification of a simple layout with large acceptance, transmission, and resolution. The design method may be easily adapted to project-specific needs. The important effects of energy degraders necessary for full fragment separator design will be addressed in a future publication.
\end{abstract}

DOI: 10.1103/PhysRevSTAB.10.064002

PACS numbers: 41.75. $-\mathrm{i}, 41.85 .-\mathrm{p}, 42.15 . \mathrm{Fr}$

\section{INTRODUCTION}

The next generation of research in nuclear physics requires advanced exotic beam facilities based on heavy-ion drivers. Over the past few years, several projects around the world evolved, and today are in various phases from preconceptual design to commissioning. Among the prominent examples, we mention the RIBF at RIKEN, Japan [1], the FAIR at GSI, Germany [2], the SPIRAL2 upgrade at GANIL, France [3], and plans for an exotic beam facility in the U.S. [4,5]. Although the parameters of the latter facility are not yet firmly settled, it is considered to be high priority for a new research facility for nuclear physics in the U.S. in the near future $[6,7]$.

The main components of a heavy-ion based exotic beam facility are the primary beam production area, driver accelerator, fragment separator, in-flight area, gas cell, postaccelerator, and various experimental areas. In this paper we present the optical design concepts developed for the fragment separator area. The function of the fragment separator is to separate the isotope of interest from the primary beam and other by-products, and deliver the same with high efficiency to the experimental areas, while containing the large beam power of the unwanted products and primary beam. Although the requirements vary somewhat depending on the specific project, the main features of fragment separators are the same for all heavy-ion based exotic beam facilities.

The production of rare isotopes via projectile fragmentation and fission of fast beams is one of the most important

*erdelyi@anl.gov methods [8]. The reaction kinematics, especially of the fission case, produce these rare isotopes over a large phase space area. The small production cross section of many isotopes of interest requires large primary beam powers and high energy. Often, the particles of interest are only a tiny fraction of all particles produced. The fragment separator should collect and transmit these, and only these, particles to the experimental areas while minimizing losses. Altogether, the next-generation high-intensity fragment separators require large acceptance, high resolution, and large aperture superconducting magnets.

It is well known that electromagnetic fields are not enough for separating isotopes, since each isotope is characterized by a given mass and charge, while the equations of motion of a charged particle in electromagnetic fields depend only on the mass to charge ratio. To this end, a piece of absorbing material, i.e., energy degrader or wedge, is inserted in the system, resulting in Z-dependent energy loss. When combined, magnetic fields and energy degraders make possible the separation of isotopes (the socalled rigidity-energy loss-rigidity separation method [9]). The design of the fragment separators can be split into two tasks: first design the basic optics without the absorber, and later fit the absorber into the existing system. This is not the only way to approach the design, but it results in a better performing system and it provides robustness with respect to errors. The inclusion of the absorber and its effect on the separator optics and performance will be presented in a later publication.

The achievement of large acceptance and high resolution is a challenge in the presence of large aperture superconducting magnets. It requires high precision treatment of 
the beam dynamics and correction of high order aberrations. Depending on the specific task, the layout of the fragment separator could be different: for the in-flight method there is a two-stage separation [10-12] where the two stages transmit isotopes along two intersecting lines in the mass-charge plane, and the isotope selected is the one on the intersection point; if the isotopes are to be stopped in a gas cell [13] after the first stage of separation, a second stage is also needed, but in this case it is used to slow down and monochromatize the isotope beam. For examples of designs of some current $[14,15]$ and next-generation fragment separators of the first type we refer to $[12,16]$. It is interesting that all functions of the system can be obtained by repetition of the same basic cell $[11,15]$. For the inflight method the cell is repeated 4 times and for the gascell method 3 times. This fact highlights the usefulness of applying symmetries to the design of fragment separators.

In this paper we present our design of a fragment separator based on several symmetries. Our approach is based on the fact that the basic cell mentioned in the previous paragraph should be a dispersive stage, which produces a high order achromat if repeated. Also, the achromat should be realized by a minimum number of magnets. Overall, we are searching for the simplest system that satisfies the baseline requirements in terms of acceptance, transmission, and resolution. In the next section, the symmetries involved will be reviewed and detailed. In Sec. III, the theory is applied to a third order achromat. In Sec. IV, the layout obtained by applying the theory is presented. We conclude with a summary in Sec. V.

\section{SYMMETRIES APPLIED TO THE DESIGN PRINCIPLE}

Any subpart of the system, or the system as a whole, can be represented by a transfer map $\mathcal{M}$, which, if applied to some initial conditions, gives the final conditions. If the initial conditions are represented by the vector of canonically conjugate (symplectic) variables $\vec{z}=(x, a, y, b, l, \delta)$, then

$$
\vec{z}_{f}=\mathcal{M}\left(\vec{z}_{i}\right)
$$

In $\vec{z}$ the $x$ and $y$ are denoting the horizontal and vertical positions of a particle, $a$ and $b$ are the dimensionless scaled momenta, $\delta$ is the relative energy dispersion, and $l$ is the time-of-flight difference of the particle relative to the reference particle up to a scaling factor. The map $\mathcal{M}$ can be Taylor expanded with respect to the trajectory of a reference particle and represented in the form [17]

$$
\begin{aligned}
z_{m, f}= & \sum_{j=1}^{6} z_{j, i}\left\{\left(z_{m, f} \mid z_{j}\right)+\frac{1}{2} \sum_{k=1}^{6} z_{k, i}\left\{\left(z_{m, f} \mid z_{j} z_{k}\right)\right.\right. \\
& \left.\left.+\frac{1}{3 !} \sum_{l=1}^{6} z_{l, i}\left\{\left(z_{m, f} \mid z_{j} z_{k} z_{l}\right)+\ldots\right\}\right\}\right\} .
\end{aligned}
$$

The coefficients $\left(z_{m} \mid z_{j}\right)$ are the elements of the (first order) transfer map, while $\left(z_{m} \mid z_{j} z_{k}\right),\left(z_{m} \mid z_{j} z_{k} z_{l}\right)$ are the aberration coefficients (appearing at second and higher orders in the transfer map). The symmetries applied to the system, as described next, have the effect of constraining the various coefficients. Often, these constraints cancel many aberrations. We note that some of the symmetries described next in general are broken by the introduction of absorbers. It will be the subject of a future publication to study to what extent the symmetries can be restored in a system with wedges.

\section{A. Time-independence "symmetry"}

No fields are explicitly time dependent in the system, which entails that the total (sum of kinetic and potential) energy is conserved. Of course, if the system is purely magnetic then the kinetic energy is conserved. This condition results in the following simplifications [17]:

$$
\begin{gathered}
\left(\ldots \mid \ldots l^{i_{l}} \ldots\right)=0, \quad i_{l}>0, \quad \operatorname{except}(l \mid l)=1, \\
(\delta \mid \ldots)=0, \quad \operatorname{except}(\delta \mid \delta)=1 .
\end{gathered}
$$

\section{B. Midplane symmetry}

Since it is a constraint of the layout considered here that all elements have $y=0$ as a symmetry plane, the motion above and below that plane must be identical. This cancels half of the aberrations, namely [17],

$$
\begin{array}{ll}
\left(x \mid x^{i_{x}} a^{i_{a}} y^{i_{y}} b^{i_{b}} l^{i_{l}} \delta^{i_{\delta}}\right)=0, & \text { if } i_{y}+i_{b} \text { is odd, } \\
\left(a \mid x^{i_{x}} a^{i_{a}} y^{i_{y}} b^{i_{b}} l^{i_{l}} \delta^{i_{\delta}}\right)=0, & \text { if } i_{y}+i_{b} \text { is odd } \\
\left(y \mid x^{i_{x}} a^{i_{a}} y^{i_{y}} b^{i_{b}} l^{i_{l}} \delta^{i_{\delta}}\right)=0, & \text { if } i_{y}+i_{b} \text { is even, } \\
\left(b \mid x^{i_{x}} a^{i_{a}} y^{i_{y}} b^{i_{b}} l^{i_{l}} \delta^{i_{\delta}}\right)=0, & \text { if } i_{y}+i_{b} \text { is even, } \\
\left(l \mid x^{i_{x}} a^{i_{a}} y^{i_{y}} b^{i_{b}} l^{i_{l}} \delta^{i_{\delta}}\right)=0, & \text { if } i_{y}+i_{b} \text { is odd, } \\
\left(\delta \mid x^{i_{x}} a^{i_{a}} y^{i_{y}} b^{i_{b}} l^{i_{l}} \delta^{i_{\delta}}\right)=0, & \text { if } i_{y}+i_{b} \text { is odd. }
\end{array}
$$

\section{Symplectic symmetry}

All Hamiltonian systems, including charged particles in electromagnetic fields, obey this dynamical symmetry of fundamental importance. If we denote the Jacobian of the transfer map of any section of the system by $M=\operatorname{Jac}(\mathcal{M})$, the mathematical expression of the symplectic symmetry is [17]

$$
M^{T} J M=J,
$$

where ${ }^{T}$ denotes the transpose of a matrix, and $J$ is a $2 n \times$ $2 n$ matrix with block form

$$
J=\left(\begin{array}{cc}
0 & I \\
-I & 0
\end{array}\right)
$$

$I$ being the $n \times n$ unit matrix. This relation imposes many interdependencies among the map elements. A set of such 
relations can be derived order by order. It has been done up to second order in [18], but due to rapidly increasing complexity the set of relations involving first, second, and third order coefficients have not been derived so far. With the help of symbolic computation programs like MATHEMATICA [19] we obtained this set of relations. Because of its length it can be found in Appendix A. These formulas are generally valid, and might be useful in general optics designs outside the scope of fragment separators.

\section{Mirror symmetry}

Basic mechanical systems, including charged particles in electromagnetic fields, possess the so-called timereversal symmetry [20]. It means that, if an initial configuration of such a system evolves forward in time to a final configuration, then the time-reversed final configuration evolves backward in time towards the time-reversed initial configuration. Time-reversed configuration means switching the direction of the motion and the sign of the time. Hence, if $\vec{z}_{f}=\mathcal{M}\left(\vec{z}_{i}\right), \mathcal{M}_{r}$ is the map of the reversed system (the same elements traversed in opposite order), and we define the time-reversal operator by $\mathcal{R}(x, a, y, b, l, \delta)=(x,-a, y,-b,-l, \delta)$, we obtain $\mathcal{M}_{r}\left(\mathcal{R}\left(\vec{z}_{f}\right)\right)=\mathcal{R}\left(\vec{z}_{i}\right)$, or, since $\vec{z}_{i}$ is arbitrary and $\mathcal{R}^{-1}=$ $\mathcal{R}$,

$$
\mathcal{M}_{r}=\mathcal{R} \circ \mathcal{M}^{-1} \circ \mathcal{R}
$$

This general formula offers a convenient way to compute the map of the reversed system, if one has a method of explicitly computing the inverse of a transfer map. Indeed, such a method exists by using differential algebraic techniques [17], and is available in codes such as COSY Infinity [21]. The algorithm is based on a fixed point iteration that converges to the exact result in finitely many steps. While much more memory and time consuming than the numerical method, the algorithm can be easily implemented in a symbolic algebra computational tool like MATHEMATICA. Reasonable memory requirements and running time restricts its practical use to low orders.

Equation (7) implies that if a system is mirror symmetric about some transverse section, the reversed system's map must be the same as the forward system's map, which entails that

$$
\mathcal{M} \circ \mathcal{R} \circ \mathcal{M}=\mathcal{R} \text {. }
$$

This relation also contains many restrictions. Up to second order, these relations can be found in Appendix B. Because of these relations, the aberrations of a mirror symmetric system cannot take arbitrary values.

This concludes the section on symmetries. Now we turn our attention to the practical use of these symmetries in fragment separator designs.

\section{APPLICATION OF THE SYMMETRIES TO DESIGN PRINCIPLES}

The isotopes of interest to be separated by the fragment separator are created from essentially a pointlike primary beam hitting a target, and the resulting emittance due to reaction kinematics is dominated by large angular divergence and energy spread. The main requirement of the fragment separator is that the final position of the particles at the separation slit should depend only on mass and charge. These conditions require a high order achromat with a large intermediate dispersion that directly translates into large mass and charge resolutions. Mathematically, a high order achromat has a transfer map that is the identity map up to the order of the achromat. There are theories of high order achromats [22], but here the goal is to find the simplest system with the minimum number of magnets and low residual aberrations. The obvious choice would be to follow a system described by some transfer map with a system that has a transfer map that is the inverse of the first one. This way, the total map would be exactly identity to all orders. Unfortunately, such a system is not available. However, the reversed system's map is almost the inverse, if it were not for the time-reversal operator. To this end, mirror symmetry can be used in the following way: assume the first half of the achromat is dispersive and described by $\mathcal{M}_{d}$. If the second half is the reversed layout of the first half, the total map $\mathcal{M}_{t}$ will be

$$
\mathcal{M}_{t}=\mathcal{R} \circ \mathcal{M}_{d}^{-1} \circ \mathcal{R} \circ \mathcal{M}_{d}
$$

Assume that $\mathcal{M}_{d}$ commutes with $\mathcal{R}$ under composition, $\left[\mathcal{M}_{d}, \mathcal{R}\right]=0$. Then,

$$
\mathcal{M}_{t}=\mathcal{R} \circ \mathcal{M}_{d}^{-1} \circ \mathcal{M}_{d} \circ \mathcal{R}=\mathcal{R} \circ \mathcal{R}=I .
$$

Therefore, if the commutator vanishes, we designed an arbitrary order achromat. Unfortunately, the commutator does not vanish in general. The explicit calculation of the commutator (assuming time independence and midplane symmetries) shows that, neglecting time-of flight effects that are of no interest here, the following aberration coefficients should vanish in $\mathcal{M}_{d}$ for the whole commutator to vanish completely:

$$
\begin{array}{ll}
\left(x \mid x^{i_{x}} a^{i_{a}} y^{i_{y}} b^{i_{b}} \delta^{i_{\delta}}\right)=0, & \text { if } i_{a}+i_{b} \text { is odd, } \\
\left(a \mid x^{i_{x}} a^{i_{a}} y^{i_{y}} b^{i_{b}} \delta^{i_{\delta}}\right)=0, & \text { if } i_{a}+i_{b} \text { is even } \\
\left(y \mid x^{i_{x}} a^{i_{a}} y^{i_{y}} b^{i_{b}} \delta^{i_{\delta}}\right)=0, & \text { if } i_{a}+i_{b} \text { is odd } \\
\left(b \mid x^{i_{x}} a^{i_{a}} y^{i_{y}} b^{i_{b}} \delta^{i_{\delta}}\right)=0, & \text { if } i_{a}+i_{b} \text { is even. }
\end{array}
$$

To first order, these entail that the first half of the system should be point-to-point imaging and parallel-to-parallel in both horizontal and vertical planes, and the final angles should be energy independent. The 5 first order conditions are augmented by 15 aberration coefficients at second order and 35 at third order. The number increases drastically at higher orders. For more details, we refer to [23]. 
At this point we know that, if we design the achromatic system such that it is mirror symmetric around the middle where the dispersion is maximized, the first half is imaging and telescopic, and some aberrations are canceled, then we obtain a high order achromat with large resolution and low residual aberrations. The first half is dispersive, so it is necessary to include one dipole. The number of quadrupoles is determined by the number of first order conditions to be satisfied. The 15 second order conditions, however, appear to be too many to satisfy.

To decrease the number of second order conditions we resort again to mirror symmetry. Since the first half can be realized with a single dipole, we pick another mirror symmetric point to be the middle of the dipole. By doing this, the whole system will have a double mirror symmetry. To show that this reduces the number of second order conditions to be met, we make explicit the constraints included in Eq. (8). The results show that many commutator elements vanish if all first order conditions are met. Specifically, if $(x \mid a)_{d}=(a \mid x)_{d}=(y \mid b)_{d}=(b \mid y)_{d}=$ $(a \mid \delta)_{d}=0$, then mirror symmetry alone about the middle of the dipole results in all second order commutator elements automatically being zeroed out except $5:(x \mid a \delta)_{d}$, $(a, x \delta)_{d},(a \mid \delta \delta)_{d},(y \mid b \delta)_{d}$, and $(b \mid y \delta)_{d}$. Moreover, the following relationships are established:

$$
\begin{aligned}
& (x \mid x)_{d}=(a \mid a)_{d}= \pm 1, \\
& (y \mid y)_{d}=(b \mid b)_{d}= \pm 1, \\
& (x \mid \delta)_{d}\left[1+(x \mid x)_{d}\right]=0, \\
& (a \mid \delta)_{d}\left[(a \mid a)_{d}-1\right]=0 .
\end{aligned}
$$

Since $(x \mid \delta)_{d} \neq 0$, it follows that $(x \mid x)_{d}=(a \mid a)_{d}=-1$ and $(a \mid \delta)_{d}=0$ automatically. This leaves 4 independent first order conditions. Some second order aberrations are not independent. The following relations are obtained:

$$
\begin{gathered}
(x \mid x \delta)_{d}+(x \mid \delta)_{d}(x \mid x x)_{d}=0, \\
2(a \mid a \delta)_{d}+(x \mid \delta)_{d}(a \mid x a)_{d}=0, \\
2(a \mid \delta \delta)_{d}+(x \mid \delta)_{d}(a \mid x \delta)_{d}=0, \\
2(y \mid y \delta)_{d}+(x \mid \delta)_{d}(y \mid x y)_{d}=0, \\
2(b \mid b \delta)_{d}+(x \mid \delta)_{d}(b \mid x b)_{d}=0 .
\end{gathered}
$$

Equation (18) shows that $(a, x \delta)_{d}$ and $(a \mid \delta \delta)_{d}$ are proportional, leaving 4 independent second order conditions to cancel the 5 second order terms listed above. The relations derived in [18] show that among the remaining 4 independent first order and 4 independent second order aberrations symplecticity does not impose any additional constraints. Therefore, we can conclude that a double mirror symmetric system that contains 4 cells, with only a half-dipole and 4 superimposed multipoles having quadrupole and sextupole components per cell, will satisfy all our requirements to second order. The number of multipoles is determined by the 4 first and second order conditions to be met. Although we found first order solutions with only three quadrupoles per cell [due to the additional symmetry of $(x \mid x)=(a \mid a)$ for all elements affecting the first order transfer matrix], the 4 second order conditions generically require 4 sextupoles per cell also arranged mirror symmetrically in the two cells. It is envisioned due to cost reasons, lack of space, and innovative design, that only superimposed multipoles will be used. Also, the 4 quadrupole solution enables more flexibility regarding the length of the drift spaces and the location of the beam dump. Hence, the number of multipoles is settled to 4 . For details of superimposed multipoles, with variable multipole components we refer to [24].

Assuming that we have satisfied all conditions for a second order achromat, in the following we consider third order effects. The number of commutator elements at third order that need to be cancelled is 35 . This is very large, so again we look for all the simplifications that might come from application of mirror and symplectic symmetries. Mirror symmetry, according to Eq. (8), gives some interesting results: 21 third order commutator elements are linked by 11 proportionality relations, reducing the effectively independent commutator elements by 11 . The following proportionality relations are obtained:

$$
\begin{aligned}
&(x \mid x x a) \propto(x \mid x a \delta),(x \mid x y b) \propto(x \mid x b \delta) \\
&(a \mid x x x) \propto(a \mid x x \delta) \propto(a \mid \delta \delta \delta),(a \mid x a a) \propto(a \mid a a \delta),(a \mid x y y) \\
& \propto(a \mid y y \delta),(a \mid x b b) \propto(a \mid b b \delta), \\
&(y \mid x a y) \propto(y \mid a y \delta),(y \mid x x b) \propto(y \mid x b \delta), \\
&(b \mid x x y) \propto(b \mid x y \delta),(b \mid x a b) \propto(b \mid a b \delta) .
\end{aligned}
$$

Additionally, mirror symmetry gives 4 second order and 4 third order proportionality relations among noncommutator elements. Moreover, it is interesting to note that mirror symmetry also implies that 20 noncommutator third order aberrations are completely determined by the fixed second order layout. Hence, no octupole may correct any of these aberrations. Specifically, these are the following: $(x \mid x x x)$, $(x \mid x a a),(x \mid x y y),(x \mid x b b),(x \mid a y b),(a \mid x x a),(a \mid x y b)$, $(a \mid a a a),(a \mid a y y),(a \mid a b b),(y \mid x x y),(y \mid x a b), \quad(y \mid a a y)$, $(y \mid y y y),(y \mid y b b),(b \mid x a y),(b \mid x x b),(b \mid a a b),(b \mid y y b)$, $(b \mid b b b)$.

Symplecticity did not help in reducing the number of independent second order commutator-aberrations (although it provides 7 proportionality relations among noncommutator second order aberrations), but the new third order relations of Appendix A give 14 new proportionality relations among third order commutator elements, if we again assume all second order conditions satisfied. These are the following: 


$$
\begin{aligned}
(x \mid x x a) & \propto(a \mid x a a),(a \mid a a \delta) \propto(x \mid x a \delta),(x \mid x y b) \propto(a \mid a y b) \\
& \propto(b \mid x a b) \propto(y \mid x a y), \\
(x \mid a y y) & \propto(b \mid a a y),(x \mid y b \delta) \propto(b \mid a b \delta) \propto(y \mid a y \delta),(a \mid x y y) \\
& \propto(b \mid x x y),(a \mid y y \delta) \propto(b \mid x y \delta), \\
(a \mid x b b) & \propto(y \mid x x b),(a \mid b b \delta) \propto(y \mid x b \delta),(x \mid a b b) \\
& \propto(y \mid a a b),(y \mid y y b) \propto(b \mid y b b) .
\end{aligned}
$$

Since there is no overlap between Eqs. (21)-(24) and Eqs. (25)-(27) due to mirror and symplectic constraints, the number of independent third order conditions is reduced to $10=35$ (total $)-[14$ (simplecticity $)+$ 11(mirror)]. Therefore, if mirror symmetry is obeyed up to third order elements, the number of octupoles per cell needed is 10. Unfortunately, this number is still too large for practical systems. Therefore, the theory shows that a perfect third order solution is not possible with a reasonable number of octupoles. This, coupled with the fact that many noncommutator third order aberrations cannot be altered by any number or placement of octupoles, suggests to break the double mirror symmetry at third order (while maintaining mirror symmetry with respect to the dispersive image).

The number of multipoles per cell is 4 according to the second order solution. If these multipoles are equipped with a variable octupole component and made independent, the reasonable number of octupoles to attempt a good third order solution is 8. Is it possible to obtain a good third order solution with 8 octupoles if 20 are needed for a perfect one? The answer is a partial yes. If we assume that the primary beam is pointlike, then all positiondependent commutator aberrations vanish (in practice these aberrations are usually small). In this approximation, the third order commutator terms are the following:

$$
\begin{gathered}
(x \mid a a a),(x \mid a b b),(x \mid a \delta \delta), \\
(a \mid a a \delta),(a \mid b b \delta),(a \mid \delta \delta \delta), \\
(y \mid a a b),(y \mid b b b),(y \mid b \delta \delta),
\end{gathered}
$$

$(b \mid a b \delta)$.

Equation (27) reduces the number of independent aberrations by one, resulting in 9 independent third order aberrations. Therefore, in practice we have 8 octupoles to minimize 9 third order aberrations. While there is generally no unique solution to this problem, in practice one can obtain quite good solutions.

Equation (7) can be used in conjunction with symbolic inversion of maps to compute the effect of not correcting aberrations (28)-(31). The results show that all aberrations change sign and double in magnitude, but no other aberrations are introduced. Therefore, if angular aberrations are neglected, a solution can be found with only 5 octupoles. However, the angular aberrations are important for the subsequent separation stages, and neglecting them might not always be a good choice. The remaining octupoles may be used to correct position-dependent aberrations, in case some coefficients are unusually large.

In the next section we present a practical realization of the optics of a fragment separator based on these symmetry theories.

\section{OPTICS OF A FRAGMENT SEPARATOR}

The double mirror symmetry of the system implies that only the specific layout of one-fourth of the system needs to be optimized, the map of the whole system being given by symmetry operations. In this cell, according to the theory, there will be 4 superimposed multipoles and a half-dipole at the end. The first order layout is determined by drifts, quadrupoles, and the dipole. Denoting the map of the system from beginning to the end of the cell, i.e., the middle of the first dipole, by $\mathcal{M}_{m}$, the map $\mathcal{M}_{d}$ up to the dispersive image can be computed utilizing Eq. (7). Hence, we obtain the conditions that the matrix elements in $\mathcal{M}_{m}$ must satisfy (skipping the time-of-flight part) in order to obey the conditions derived in the previous section. Specifically, we obtain the following first order transfer matrix:

$$
\begin{gathered}
M_{d}=\left(\begin{array}{ccc}
(a \mid x)_{m}(x \mid a)_{m}+(a \mid a)_{m}(x \mid x)_{m} & 2(a \mid a)_{m}(x \mid a)_{m} \\
2(a \mid x)_{m}(x \mid x)_{m} & (a \mid x)_{m}(x \mid a)_{m}+(a \mid a)_{m}(x \mid x)_{m} & \\
0 & 0 & \\
0 & 0 & 2(a \mid \delta)_{m}(x \mid a)_{m} \\
0 & 0 & 2(a \mid \delta)_{m}(x \mid x)_{m} \\
0 & 0 & 0 \\
0 & 0 & 0 \\
(b \mid y)_{m}(y \mid b)_{m}+(b \mid b)_{m}(y \mid y)_{m} & 2(b \mid b)_{m}(y \mid b)_{m} & 1 \\
2(b \mid y)_{m}(y \mid y)_{m} & (b \mid y)_{m}(y \mid b)_{m}+(b \mid b)_{m}(y \mid y)_{m} & 0 \\
0 & &
\end{array}\right),
\end{gathered}
$$




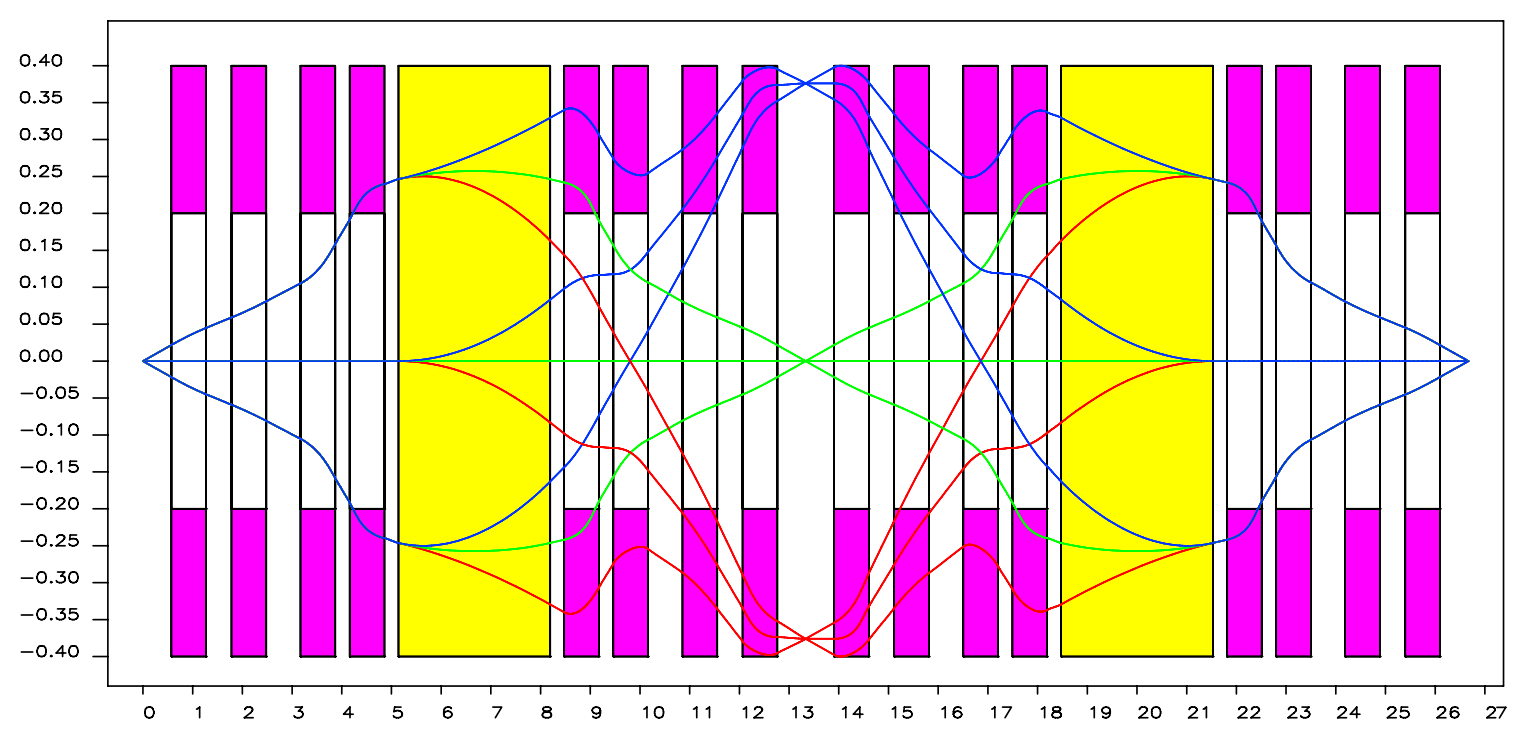

FIG. 1. (Color) First order horizontal beam envelope corresponding to an initial beam emittance of $\pm 1 \mathrm{~mm}, \pm 50 \mathrm{mrad}$, and $\pm 16 \%$ energy dispersion. Yellow (light) boxes represent dipoles and the magenta (dark) boxes represent multipoles with appropriate apertures. The multipoles have two apertures: the larger (colored) represent the horizontal while the smaller (inner, white) represent the vertical apertures.

where the subscript $m$ denotes matrix elements at the middle of the first dipole. Looking at the horizontal submatrix, we notice that $(x \mid \delta)_{d}=2(a \mid \delta)_{m}(x \mid a)_{m}$ and $(a \mid \delta)_{d}=2(a \mid \delta)_{m}(x \mid x)_{m}$. The conditions $(x \mid \delta)_{d} \neq 0$ and $(a \mid \delta)_{d}=0$ imply $(x \mid a)_{m} \neq 0$ and $(x \mid x)_{m}=0$, since $(a \mid \delta)_{m} \neq 0$ because it is created only by the half-dipole at the end of the cell. To maximize resolution, $(x \mid \delta)_{d}$ needs to be maximized, which in turn implies that $(x \mid a)_{m}$ must be maximized. Therefore, the beam size in the dipole must be maximized. Essentially, this is just another proof of the well known fact that the resolution depends on the magnetic flux enclosed by the ray with the largest divergence in the dipole. The telescopic imaging conditions at the dispersive image require $(a \mid a)_{m}(x \mid a)_{m}=0$ and $(a \mid x)_{m} \times$ $(x \mid x)_{m}=0$. Hence we immediately obtain $(a \mid a)_{m}=0$. In the vertical plane we need $(b \mid b)_{m}(y \mid b)_{m}=(b \mid y)_{m}(y \mid y)_{m}=$ 0 . These two conditions can be satisfied simultaneously if $(y \mid y)_{m}=(b \mid b)_{m}=0$ or $(y \mid b)_{m}=(b \mid y)_{m}=0$. Other combinations are forbidden by the symplectic condition. In summary, the fundamental cell must be point-to-parallel

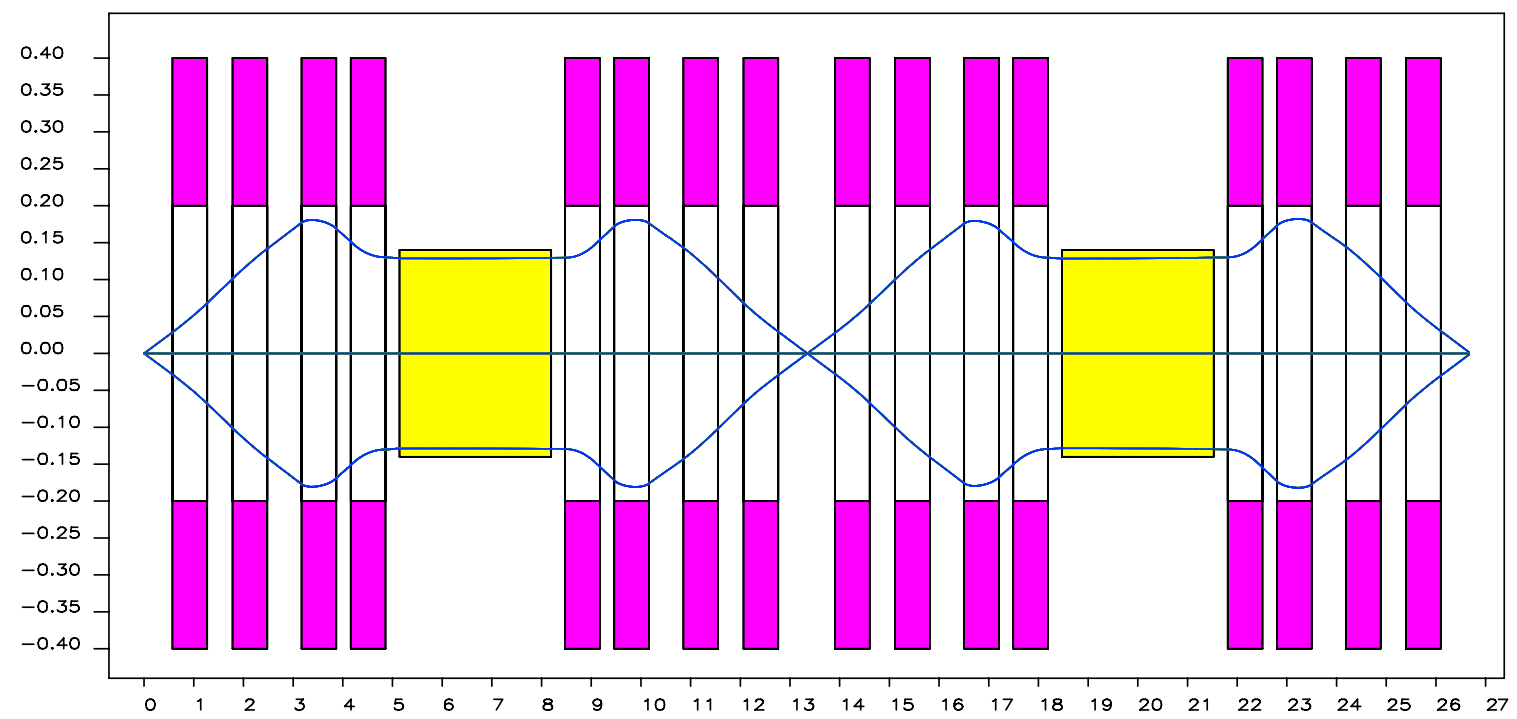

FIG. 2. (Color) First order vertical beam envelope corresponding to an initial beam emittance of $\pm 1 \mathrm{~mm}, \pm 50 \mathrm{mrad}$, and $\pm 16 \%$ energy dispersion. Yellow (light) boxes represent dipoles and the magenta (dark) boxes represent multipoles with appropriate apertures. The multipoles have two apertures: the larger (colored) represent the horizontal while the smaller (inner, white) represent the vertical apertures. 
and parallel-to point horizontally, and there is a choice of being point-to-parallel and parallel-to point, or point-topoint and parallel-to-parallel vertically.

At this point the specific layout, subject to all conditions derived above, must be obtained using numerical optimization. Most standard beam optical codes do not allow constrained optimization. In order to avoid nonphysical results such as negative drifts or too large pole tip fields, a simple MATHEMATICA notebook was implemented to do a first order constrained optimization. The optimization parameters were all the drift lengths and quadrupole strengths. The parameters of the dipole were fixed to reasonable values. Constraints were all drift lengths (to be larger than a minimum, imposed mainly due to engineering concerns) and a maximum pole tip field in the multipoles, along with maximum values for magnet apertures. As figures of merit we maximized resolution while minimizing the maximum beam size anywhere in the system, and minimizing the quadrupole strengths in order to limit the size of higher order aberrations. The best system resulting from an extensive set of optimization runs over the full spectrum of parameter space is presented in Figs. 1 and 2. For more details see [23]. The figures show 4 cells, i.e., the full achromat, and the horizontal and vertical beam envelopes. The horizontal multipole aperture is $\pm 40 \mathrm{~cm}$, the vertical aperture is $\pm 20 \mathrm{~cm}$ (assuming rectangular cross sections), the multipole length is $70 \mathrm{~cm}$, while the vertical dipole gap is $\pm 12 \mathrm{~cm}$. The total length of the system is $27 \mathrm{~m}$. In the example shown the beam rigidity is $8 \mathrm{Tm}$. For this value of the magnetic rigidity the maximum quadrupole pole tip field is $3 \mathrm{~T}$. Most of the multipole strengths are small. Parameters of the dipole are $5 \mathrm{~m}$ radius and $35^{\circ}$ angle. All drift lengths are at least $25 \mathrm{~cm}$. The parameter list is contained in Table I.

For higher order designs the code COSY Infinity was used [21]. The first order input was supplied by the MATHEMATICA results. Since the number and location of the sextupoles and octupoles was fixed by the theory and practical considerations, a numerical optimization found the strengths that were needed to satisfy the requirements derived in the theory section. Some generic fringe field effects were taken into account. These end effects modified only slightly the magnet strengths. This is not surprising since the theory is valid for any complicated end effects, including overlapping fringe fields, as long as the system stays symmetric. Figures 3 and 4 show the third order acceptance of the fragment separator. The resolution is maximized by maximizing the dispersion, and all aberrations are below $1 \mathrm{~mm}$ in magnitude.

One can notice that at the dispersive image plane some aberrations are still present. This is mainly due to three uncorrected noncommutator aberrations: $(x \mid a a)_{d},(x \mid b b)_{d}$, and $(y \mid a b)$. Simplecticity implies $(x \mid b b)_{d} \propto(y \mid a b)_{d}$. Hence, there are two independent aberrations that spoil the second order imaging at the dispersive image. These
TABLE I. Parameter list for the case of a beam of $8 \mathrm{Tm}$ rigidity. The drifts, quadrupoles, and sextupoles are for one cell only in the order they appear in the beam line (from left to right in the figures). The 8 octupoles are from the first two cells in the order they appear in the beam line. The remaining elements are given by the symmetries detailed in Sec. IV. Some generic fringe fields are taken into account. The numerical values of the magnet strengths depend on the specific fringe field model applied. We note that, due to the large strengths of the first two octupoles, the octupole windings might be on a smaller bore than the rest of the coils since the beam size is small at the location of the respective multipoles. Also, work in progress on more advanced optimization methods might result in solutions with reduced octupole strengths.

\begin{tabular}{ccr}
\hline \hline Elements & $\begin{array}{c}\text { Pole tip field } \\
\text { at } 20 \mathrm{~cm}(T)\end{array}$ & $\begin{array}{r}\text { Pole tip field } \\
\text { at } 40 \mathrm{~cm}(T)\end{array}$ \\
\hline Quadrupoles & & \\
Q1 & 0.74 & 1.47 \\
Q2 & -0.28 & -0.55 \\
Q3 & -1.52 & -3.03 \\
Q4 & 1.10 & 2.20 \\
Sextupoles & & \\
\hline S1 & 0.02 & 0.09 \\
S2 & 0.03 & 0.12 \\
S3 & -0.29 & -1.16 \\
S4 & 0.23 & 0.94 \\
Octupoles & & -4.83 \\
\hline O1 & -0.60 & -4.75 \\
O2 & -0.60 & -0.28 \\
O3 & -0.03 & 0.64 \\
O4 & 0.08 & -0.46 \\
O5 & -0.06 & 0.78 \\
O6 & 0.10 & -0.08 \\
O7 & -0.01 & -0.01 \\
O8 & -0.001 & \\
Drifts & 57 & \\
\hline L1 & 51 & \\
L2 & 28 & \\
L3 & & \\
L4 & & \\
L5 & & \\
\hline \hline
\end{tabular}

could be corrected by two additional sextupoles if needed. The energy degrader is placed at the dispersive image plane. If the optical effects of the wedge require, this additional correction can be easily done. The wedgerelated problems will be the subject of future studies.

Another solution is possible if there is only one separation stage. In this situation the angular aberrations at the achromatic image plane are not important. Explicit calculation based on (7) shows that the two angular commutator aberrations $(a \mid x \delta)_{d}$ and $(b \mid y \delta)_{d}$ contribute only to angular aberrations at the achromatic image. Therefore, one can choose to correct with the 4 sextupoles available $(x \mid a a)_{d}$, 


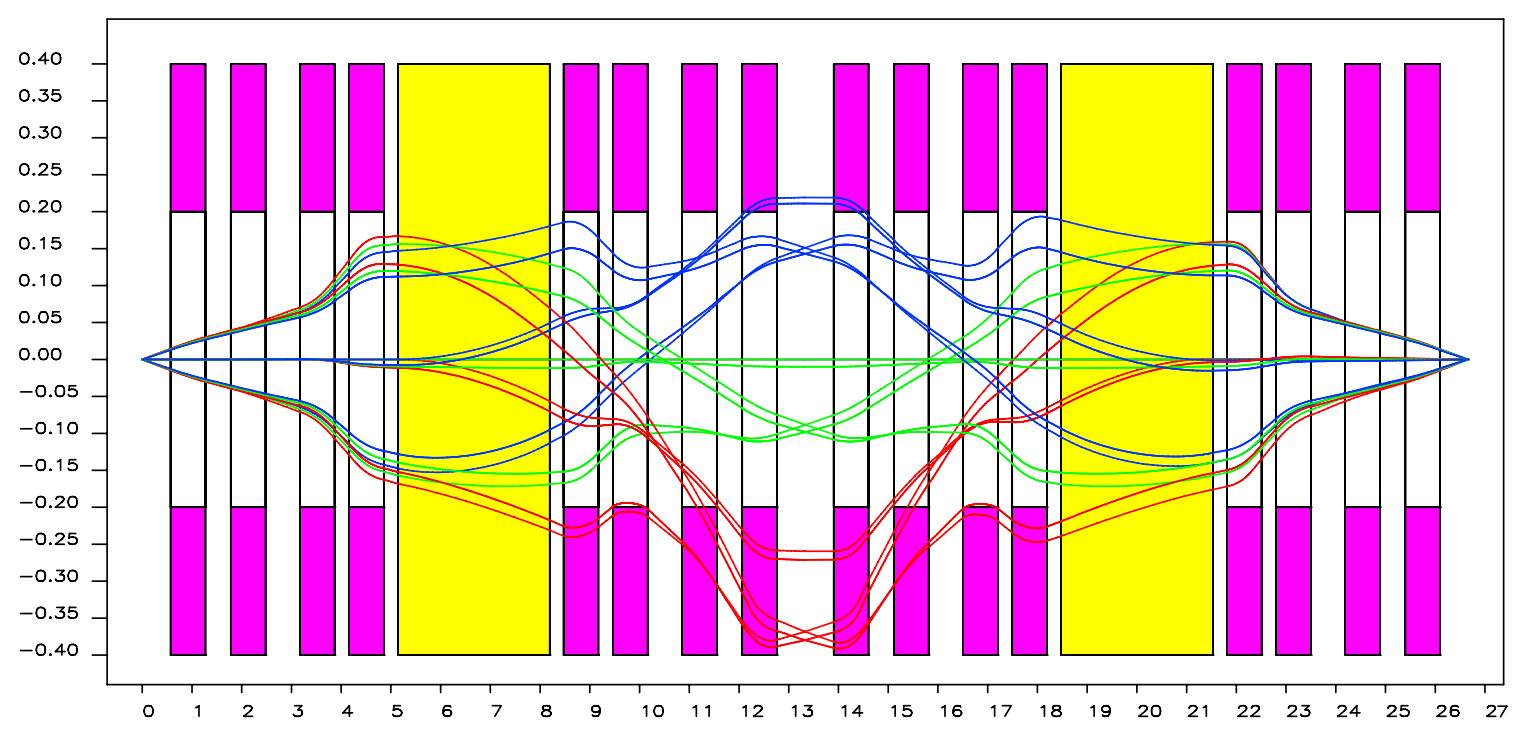

FIG. 3. (Color) Third order horizontal envelope corresponding to acceptance (initial beam emittance of $\pm 1 \mathrm{~mm}$, $\pm 40 \mathrm{mrad}$ horizontally and $\pm 50 \mathrm{mrad}$ vertically, and $\pm 10 \%$ energy dispersion).

$(x \mid b b)_{d},(x \mid a \delta)_{d}$, and $(y \mid b \delta)_{d}$. This way the dispersive image stays imaging and at the achromatic image plane all positional aberrations stay small. An example of such a solution is shown in Fig. 5. However, for the two-stage separation the large angular aberrations become detrimental in the second stage of separation. In this case it is worthwhile to choose the 6 sextupole solution described in the preceding paragraph.

Preliminary studies indicate that the symmetries are beneficial even if random errors are present in the system. Some properties of the symmetric layout survive if the errors are not too large. One important factor determining the quality of the system is the magnitude of the residual aberrations. In this aspect the solution shown in Figs. 1-4 is far superior to all alternatives mentioned and studied. Figure 6 shows the fifth order acceptance, a good measure of the residual aberrations. All alternate designs gave poor results in this respect. Again, for details we refer the reader to [23].

For a quantitative measure of what was achieved with the system, Table II shows some values of the percentage of particles transmitted by the system using realistic initial distributions supplied by the code MCNPX for fission of ${ }^{238} \mathrm{U}$ [25]. The transmission is essentially determined by

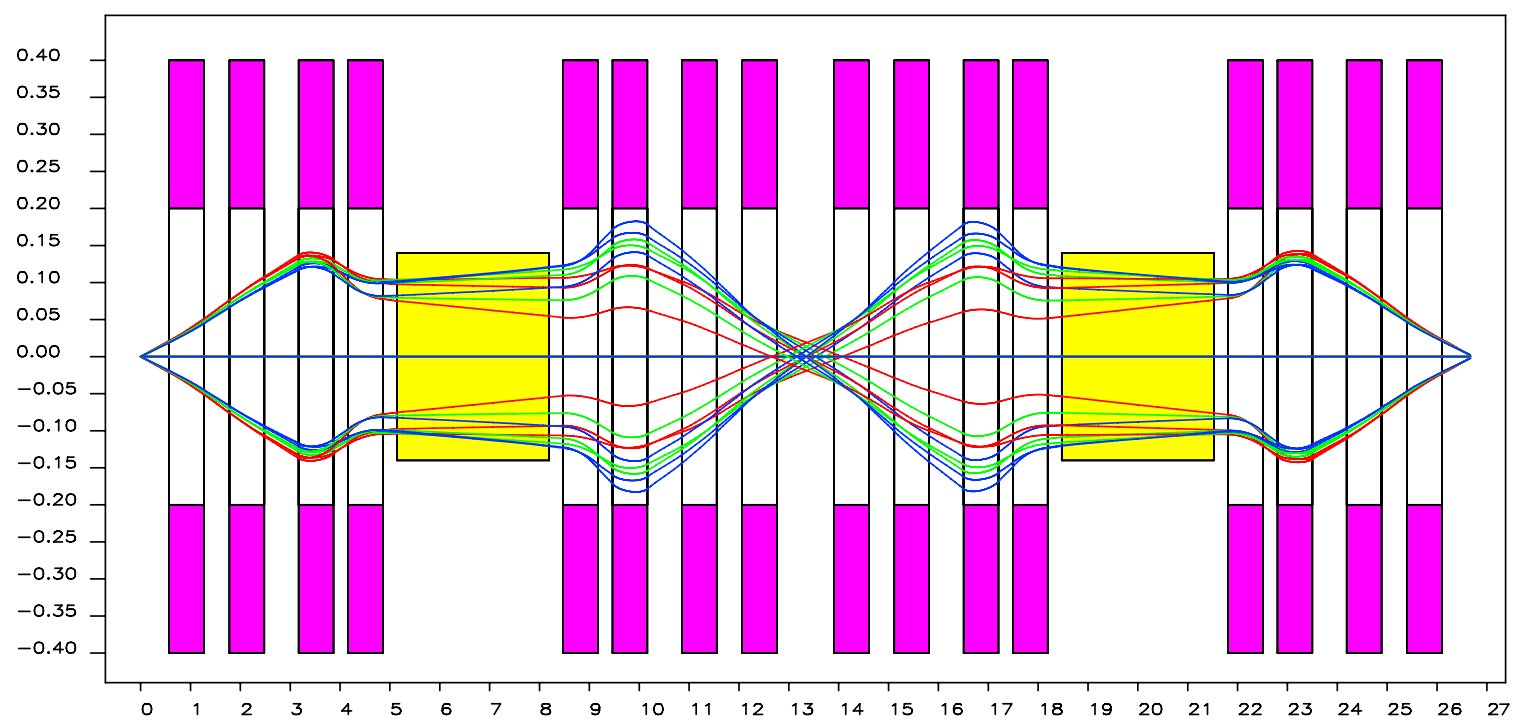

FIG. 4. (Color) Third order vertical envelope corresponding to acceptance (initial beam emittance of $\pm 1 \mathrm{~mm}, \pm 40 \mathrm{mrad}$ horizontally and $\pm 50 \mathrm{mrad}$ vertically, and $\pm 10 \%$ energy dispersion). 


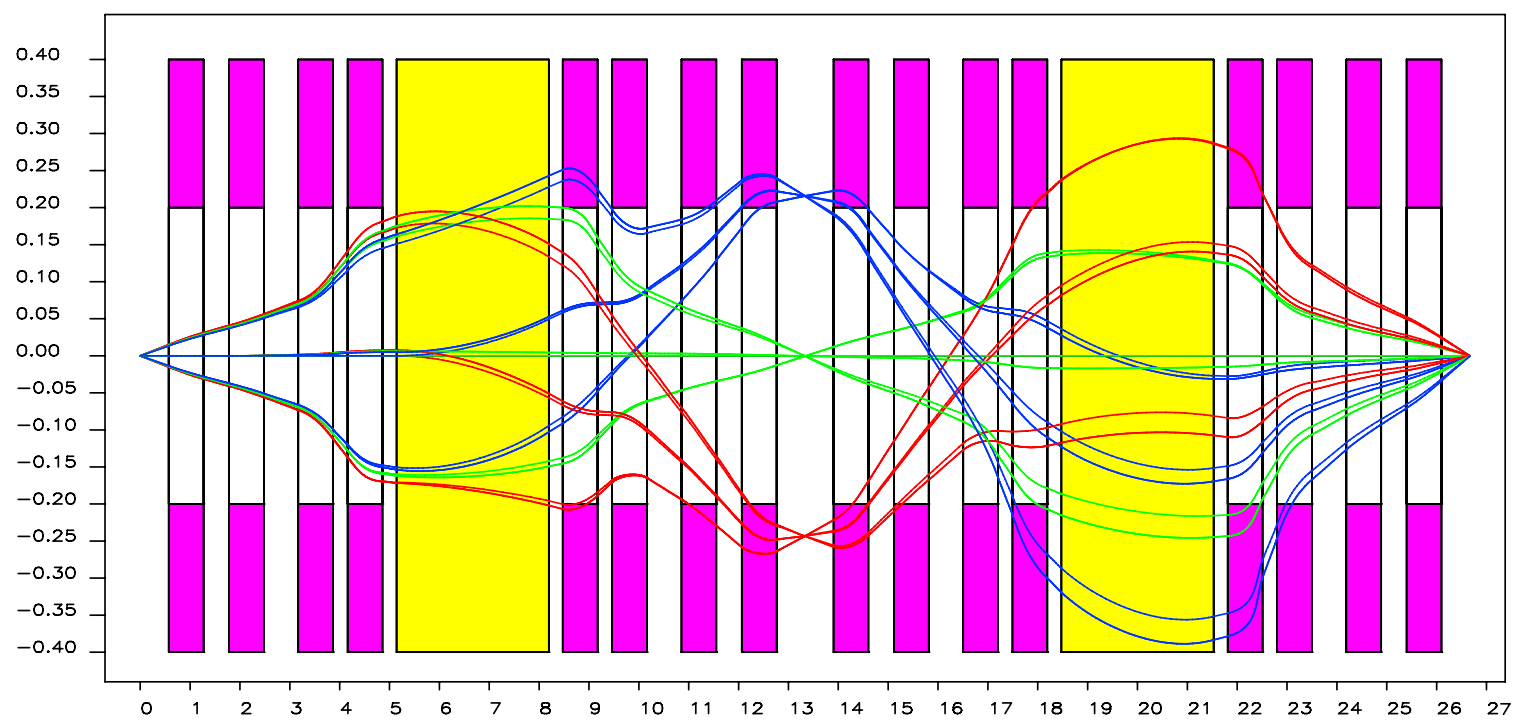

FIG. 5. (Color) Alternate design, which cancels the positional aberrations at the dispersive plane at the detriment of some angular aberrations.

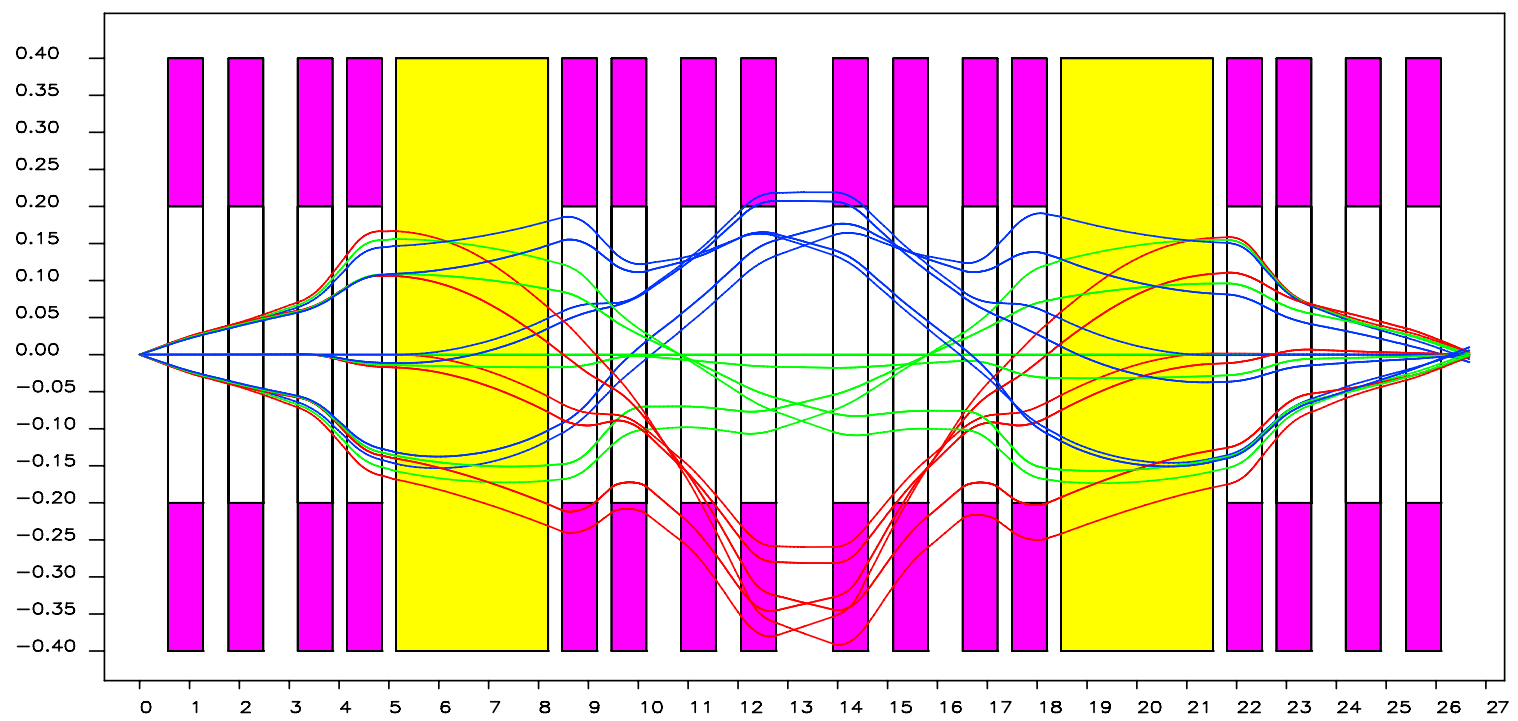

FIG. 6. (Color) Fifth order horizontal envelope corresponding to acceptance (initial beam emittance of $\pm 1 \mathrm{~mm}$, $\pm 25 \mathrm{mrad}$ horizontally and $\pm 50 \mathrm{mrad}$ vertically, and $\pm 10 \%$ energy dispersion) of the solution shown in Figs. 1-4.

the third order layout. The values shown in the table do not change significantly if the system is run at higher orders. Another figure of merit is the standard deviation of the horizontal beam size at the achromatic image. It determines the deterioration of the resolution due to higher order effects. Figure 7 shows the values of $\sigma_{x}$ computed at different orders, before and after the corrections, for an initial beam distribution that corresponds to the linear acceptance shown in Figs. 1 and 2. The third order corrections recover the linear beam size, while most particles that contribute to the larger beam sizes at fourth and higher orders are lost before reaching the achromatic image, hence not deteriorating much the resolution.
TABLE II. Transmission of ${ }^{132} \mathrm{Sn}$ and ${ }^{90} \mathrm{Rb}$ obtained from fission of ${ }^{238} \mathrm{U}$. The acceptance of the system is large enough to fit in the kinematics of $\mathrm{Sn}$ at $400 \mathrm{MeV} / u$ and it decreases with energy and mass.

\begin{tabular}{lcccc}
\hline \hline Isotope & $\begin{array}{c}\text { Energy } \\
{[\mathrm{MeV} / u]}\end{array}$ & $\begin{array}{c}\text { First order } \\
{[\%]}\end{array}$ & $\begin{array}{c}\text { Second order } \\
{[\%]}\end{array}$ & $\begin{array}{c}\text { Third order } \\
{[\%]}\end{array}$ \\
\hline${ }^{132} \mathrm{Sn}$ & 400 & 100 & 100 & 100 \\
${ }^{132} \mathrm{Sn}$ & 200 & 100 & 83 & 75 \\
${ }^{90} \mathrm{Rb}$ & 400 & 100 & 87 & 82 \\
${ }^{90} \mathrm{Rb}$ & 200 & 72 & 43 & 42 \\
\hline \hline
\end{tabular}




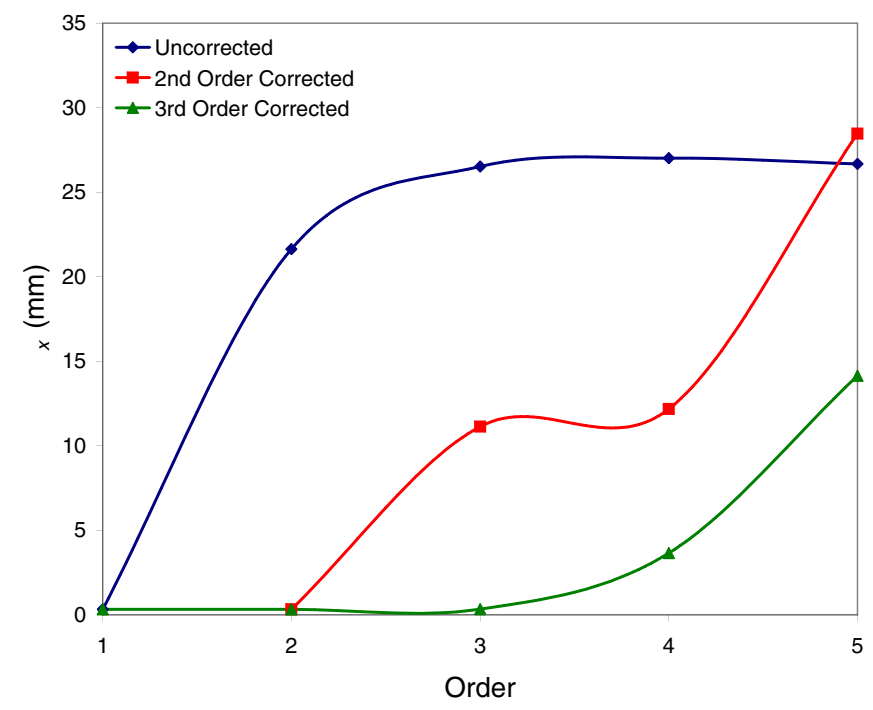

FIG. 7. (Color) Standard deviation of the horizontal beam size evaluated at different orders before and after aberration corrections.

\section{SUMMARY AND CONCLUSIONS}

We have shown that symmetries provide a powerful tool in the conceptual design of fragment separator optics and related charged particle optical devices. We derived many relationships of general interest stemming from symplectic and mirror symmetries, which might prove useful for generic optical design. The combination of analytical and numerical computation and optimization lead to the layout of a fragment separator that is a good starting point for studies of the full separator, including energy degraders [26].

We concluded that the separator can be obtained by applying symmetry operations to a basic cell that is point-to-parallel and parallel-to-point both horizontally and vertically. This cell, in turn, has a minimum number of magnets and some other useful properties. The acceptance, resolution, and transmission were optimized, taking into account the large aberrations induced by the large aperture superconducting magnets. Correction of higher order aberrations, coupling between different orders, mag- nitude of residual aberrations, and the number of magnets are all minimized by preservation of symmetries. Accordingly, the beam size after the corrections is essentially equal to the linear beam size up to third order. Fifth and higher order effects do increase the beam size, but the particles with those large values of the horizontal coordinate are lost before reaching the achromatic image, thus decreasing the transmission. This is achieved with a 4-cell system containing 4 superimposed multipoles and half a dipole per cell. The fragment separator can accept the full kinematics of a ${ }^{132} \mathrm{Sn}$ beam obtained from fission of a $400 \mathrm{MeV} / u{ }^{238} \mathrm{U}$ primary beam, but for lighter species and lower energies, due to the reaction kinematics, the transmission is less than $100 \%$. Also, random errors in the system have less of an effect as long as symmetries are maintained. This is true to some extent even for complicated fringe fields, including overlapping fields. The qualitative conclusions remain unaffected. Ongoing work related to accurate fringe field computation for the rectangular aperture multipoles envisioned for the fragment separator might slightly change some of the quantitative predictions. Other work in progress includes the effects of material-beam interactions in the design. In summary, we have provided a symmetry-based approach to a nextgeneration high-intensity large acceptance and high resolution fragment separator optics for a future exotic beam facility that can be easily adapted to specific scenarios.

\section{ACKNOWLEDGMENTS}

We would like to thank Joshua Brady (Washington University) for his work on Appendix A and Teresa Barlow for preliminary modeling work with COSY. This work was supported by the U.S. Department of Energy, Office of Nuclear Physics, under Contract No. DE-AC02$06 \mathrm{CH} 11357$.

\section{APPENDIX A: RELATIONS DUE TO SIMPLECTICITY}

Relations between aberration coefficients up to third order due to symplecticity are the following:

$$
\begin{array}{r}
-2(a \mid x x)(x \mid x a)+2(a \mid x a)(x \mid x x)+(a \mid x x a)(x \mid x)-(a \mid x)(x \mid x x a)-(a \mid x x x)(x \mid a)+(a \mid a)(x \mid x x x)=0 \\
-2(a \mid x x)(x \mid a a)+2(a \mid a a)(x \mid x x)+(a \mid x a a)(x \mid x)-(a \mid x)(x \mid x a a)-(a \mid x x a)(x \mid a)+(a \mid a)(x \mid x x a)=0 \\
2(a \mid a \delta)(x \mid x x)-2(a \mid x x)(x \mid a \delta)+(a \mid x a \delta)(x \mid x)-(a \mid x)(x \mid x a \delta)-(a \mid x x \delta)(x \mid a)+(a \mid a)(x \mid x x \delta)=0 \\
-(a \mid x)(x \mid x a a)+(a \mid x a a)(x \mid x)-(a \mid x x a)(x \mid a)+(a \mid a)(x \mid x x a)=0 \\
-2(a \mid x a)(x \mid a a)+2(a \mid a a)(x \mid x a)-(a \mid x)(x \mid a a a)+(a \mid a a a)(x \mid x)-(a \mid x a a)(x \mid a)+(a \mid a)(x \mid x a a)=0 \\
2(a \mid a \delta)(x \mid x a)-2(a \mid x a)(x \mid a \delta)-(a \mid x)(x \mid a a \delta)+(a \mid a a \delta)(x \mid x)-(a \mid x a \delta)(x \mid a)+(a \mid a)(x \mid x a \delta)=0
\end{array}
$$




$$
\begin{aligned}
& -2(a \mid x \delta)(x \mid x a)+2(a \mid x a)(x \mid x \delta)+(a \mid x a \delta)(x \mid x)-(a \mid x)(x \mid x a \delta)-(a \mid x x \delta)(x \mid a)+(a \mid a)(x \mid x x \delta)=0 \\
& -2(a \mid x \delta)(x \mid a a)+2(a \mid a a)(x \mid x \delta)+(a \mid a a \delta)(x \mid x)-(a \mid x)(x \mid a a \delta)-(a \mid x a \delta)(x \mid a)+(a \mid a)(x \mid x a \delta)=0 \\
& 2(a \mid a \delta)(x \mid x \delta)-2(a \mid x \delta)(x \mid a \delta)+(a \mid a \delta \delta)(x \mid x)-(a \mid x)(x \mid \delta \delta a)-(a \mid x \delta \delta)(x \mid a)+(a \mid a)(x \mid x \delta \delta)=0 \\
& 2(b \mid a y)(y \mid x y)-2(b \mid x y)(y \mid a y)+(a \mid a y y)(x \mid x)-(a \mid x)(x \mid a y y)-(a \mid x y y)(x \mid a)+(a \mid a)(x \mid x y y)=0 \\
& -2(b \mid x y)(y \mid a b)+2(b \mid a b)(y \mid x y)+(a \mid a y b)(x \mid x)-(a \mid x)(x \mid a y b)-(a \mid x y b)(x \mid a)+(a \mid a)(x \mid x y b)=0 \\
& 2(b \mid a y)(y \mid x b)-2(b \mid x b)(y \mid a y)-(a \mid x)(x \mid a y b)+(a \mid a y b)(x \mid x)-(a \mid x y b)(x \mid a)+(a \mid a)(x \mid x y b)=0 \\
& -2(b \mid x b)(y \mid a b)+2(b \mid a b)(y \mid x b)-(a \mid x)(x \mid a b b)+(a \mid a b b)(x \mid x)-(a \mid x b b)(x \mid a)+(a \mid a)(x \mid x b b)=0 \\
& -2(b \mid x y)(y \mid a y)+2(b \mid a y)(y \mid x y)-(b \mid y)(y \mid x a y)+(b \mid x a y)(y \mid y)-(a \mid x y y)(x \mid a)+(a \mid a)(x \mid x y y)=0 \\
& -2(b \mid x y)(y \mid a b)+2(b \mid a b)(y \mid x y)-(b \mid y)(y \mid x a b)+(b \mid x a b)(y \mid y)-(a \mid x y b)(x \mid a)+(a \mid a)(x \mid x y b)=0 \\
& -(b \mid y)(y \mid a a y)+(b \mid a a y)(y \mid y)-(a \mid a y y)(x \mid a)+(a \mid a)(x \mid a y y)=0 \\
& -2(b \mid a y)(y \mid a b)+2(b \mid a b)(y \mid a y)-(b \mid y)(y \mid a a b)+(b \mid a a b)(y \mid y)-(a \mid a y b)(x \mid a)+(a \mid a)(x \mid a y b)=0 \\
& -2(b \mid y \delta)(y \mid a y)+2(b \mid a y)(y \mid y \delta)+(b \mid a y \delta)(y \mid y)-(b \mid y)(y \mid a y \delta)-(a \mid y y \delta)(x \mid a)+(a \mid a)(x \mid y y \delta)=0 \\
& -2(b \mid y \delta)(y \mid a b)+2(b \mid a b)(y \mid y \delta)+(b \mid a b \delta)(y \mid y)-(b \mid y)(y \mid a b \delta)-(a \mid y b \delta)(x \mid a)+(a \mid a)(x \mid y b \delta)=0 \\
& -2(a \mid y y)(x \mid x a)+2(a \mid x a)(x \mid y y)+(b \mid x a y)(y \mid y)-(b \mid y)(y \mid x a y)-(a \mid x y y)(x \mid a)+(a \mid a)(x \mid x y y)=0 \\
& -2(a \mid y y)(x \mid a a)+2(a \mid a a)(x \mid y y)+(b \mid a a y)(y \mid y)-(b \mid y)(y \mid a a y)-(a \mid a y y)(x \mid a)+(a \mid a)(x \mid a y y)=0 \\
& 2(a \mid a \delta)(x \mid y y)-2(a \mid y y)(x \mid a \delta)+(b \mid a y \delta)(y \mid y)-(b \mid y)(y \mid a y \delta)-(a \mid y y \delta)(x \mid a)+(a \mid a)(x \mid y y \delta)=0 \\
& -2(a \mid y b)(x \mid x a)+2(a \mid x a)(x \mid y b)-(b \mid y)(y \mid x a b)+(b \mid x a b)(y \mid y)-(a \mid x y b)(x \mid a)+(a \mid a)(x \mid x y b)=0 \\
& -2(a \mid y b)(x \mid x a)+2(a \mid x a)(x \mid y b)-(b \mid y)(y \mid x a b)+(b \mid x a b)(y \mid y)-(a \mid x y b)(x \mid a)+(a \mid a)(x \mid x y b)=0 \\
& -2(a \mid y b)(x \mid a a)+2(a \mid a a)(x \mid y b)-(b \mid y)(y \mid a a b)+(b \mid a a b)(y \mid y)-(a \mid a y b)(x \mid a)+(a \mid a)(x \mid a y b)=0 \\
& 2(a \mid a \delta)(x \mid y b)-2(a \mid y b)(x \mid a \delta)-(b \mid y)(y \mid a b \delta)+(b \mid a b \delta)(y \mid y)-(a \mid y b \delta)(x \mid a)+(a \mid a)(x \mid y b \delta)=0 \\
& 2(a \mid y y)(x \mid x x)-2(a \mid x x)(x \mid y y)+(a \mid x y y)(x \mid x)-(a \mid x)(x \mid x y y)+(b \mid y)(y \mid x x y)-(b \mid x x y)(y \mid y)=0 \\
& -2(a \mid x x)(x \mid y b)+2(a \mid y b)(x \mid x x)+(a \mid x y b)(x \mid x)-(a \mid x)(x \mid x y b)+(b \mid y)(y \mid x x b)-(b \mid x x b)(y \mid y)=0 \\
& 2(a \mid y y)(x \mid x a)-2(a \mid x a)(x \mid y y)-(a \mid x)(x \mid a y y)+(a \mid a y y)(x \mid x)+(b \mid y)(y \mid x a y)-(b \mid x a y)(y \mid y)=0 \\
& -2(a \mid x a)(x \mid b y)+2(a \mid b y)(x \mid x a)-(a \mid x)(x \mid a y b)+(a \mid a y b)(x \mid x)+(b \mid y)(y \mid x a b)-(b \mid x a b)(y \mid y)=0 \\
& 2(a \mid y y)(x \mid x \delta)-2(a \mid x \delta)(x \mid y y)+(a \mid y y \delta)(x \mid x)-(a \mid x)(x \mid y y \delta)+(b \mid y)(y \mid x y \delta)-(b \mid x y \delta)(y \mid y)=0 \\
& -2(a \mid x \delta)(x \mid y b)+2(a \mid y b)(x \mid x \delta)+(a \mid y b \delta)(x \mid x)-(a \mid x)(x \mid y b \delta)+(b \mid y)(y \mid x b \delta)-(b \mid x b \delta)(y \mid y)=0
\end{aligned}
$$




$$
\begin{aligned}
& 2(a \mid y b)(x \mid x x)-2(a \mid x x)(x \mid y b)+(a \mid x y b)(x \mid x)-(a \mid x)(x \mid x y b)-(b \mid x x y)(y \mid b)+(b \mid b)(y \mid x x y)=0 \\
& -2(a \mid x x)(x \mid b b)+2(a \mid b b)(x \mid x x)+(a \mid x b b)(x \mid x)-(a \mid x)(x \mid x b b)-(b \mid x x b)(y \mid b)+(b \mid b)(y \mid x x b)=0 \\
& 2(a \mid y b)(x \mid x a)-2(a \mid x a)(x \mid y b)-(a \mid x)(x \mid a y b)+(a \mid a y b)(x \mid x)-(b \mid x a y)(y \mid b)+(b \mid b)(y \mid x a y)=0 \\
& -2(a \mid x a)(x \mid b b)+2(a \mid b b)(x \mid x a)-(a \mid x)(x \mid a b b)+(a \mid a b b)(x \mid x)-(b \mid x a b)(y \mid b)+(b \mid b)(y \mid x a b)=0 \\
& 2(a \mid y b)(x \mid x \delta)-2(a \mid x \delta)(x \mid y b)+(a \mid y b \delta)(x \mid x)-(a \mid x)(x \mid y b \delta)-(b \mid x y \delta)(y \mid b)+(b \mid b)(y \mid x y \delta)=0 \\
& -2(a \mid x \delta)(x \mid b b)+2(a \mid b b)(x \mid x \delta)+(a \mid b b \delta)(x \mid x)-(a \mid x)(x \mid b b \delta)-(b \mid x b \delta)(y \mid b)+(b \mid b)(y \mid x b \delta)=0 \\
& 2(a \mid y b)(x \mid x a)-2(a \mid x a)(x \mid y b)+(a \mid x y b)(x \mid a)-(a \mid a)(x \mid x y b)+(b \mid b)(y \mid x a y)-(b \mid x a y)(y \mid b)=0 \\
& 2(a \mid b b)(x \mid x a)-2(a \mid x a)(x \mid b b)+(a \mid x b b)(x \mid a)-(a \mid a)(x \mid x b b)+(b \mid b)(y \mid x a b)-(b \mid x a b)(y \mid b)=0 \\
& 2(a \mid y b)(x \mid a a)-2(a \mid a a)(x \mid y b)+(a \mid a y b)(x \mid a)-(a \mid a)(x \mid a y b)+(b \mid b)(y \mid a a y)-(b \mid a a y)(y \mid b)=0 \\
& 2(a \mid b b)(x \mid a a)-2(a \mid a a)(x \mid b b)+(a \mid a b b)(x \mid a)-(a \mid a)(x \mid a b b)+(b \mid b)(y \mid a a b)-(b \mid a a b)(y \mid b)=0 \\
& 2(a \mid y b)(x \mid a \delta)-2(a \mid a \delta)(x \mid y b)+(a \mid y b \delta)(x \mid a)-(a \mid a)(x \mid y b \delta)+(b \mid b)(y \mid a y \delta)-(b \mid a y \delta)(y \mid b)=0 \\
& 2(a \mid b b)(x \mid a \delta)-2(a \mid a \delta)(x \mid b b)+(a \mid b b \delta)(x \mid a)-(a \mid a)(x \mid b b \delta)+(b \mid b)(y \mid a b \delta)-(b \mid a b \delta)(y \mid b)=0 \\
& (a \mid x y y)(x \mid x)-(a \mid x)(x \mid x y y)+(b \mid y)(y \mid x x y)-(b \mid x x y)(y \mid y)=0 \\
& -2(b \mid x y)(y \mid a y)+2(b \mid a y)(y \mid x y)+(a \mid a y y)(x \mid x)-(a \mid x)(x \mid a y y)+(b \mid y)(y \mid x a y)-(b \mid x a y)(y \mid y)=0 \\
& 2(b \mid y \delta)(y \mid x y)-2(b \mid x y)(y \mid y \delta)+(a \mid y y \delta)(x \mid x)-(a \mid x)(x \mid y y \delta)+(b \mid y)(y \mid x y \delta)-(b \mid x y \delta)(y \mid y)=0 \\
& 2(b \mid x y)(y \mid x b)-2(b \mid x b)(y \mid x y)+-(a \mid x)(x \mid x y b)+(a \mid x y b)(x \mid x)+(b \mid y)(y \mid x x b)-(b \mid x x b)(y \mid y)=0 \\
& -2(b \mid x b)(y \mid a y)+2(b \mid a y)(y \mid x b)-(a \mid x)(x \mid a y b)+(a \mid a y b)(x \mid x)+(b \mid y)(y \mid x a b)-(b \mid x a b)(y \mid y)=0 \\
& 2(b \mid y \delta)(y \mid x b)-2(b \mid x b)(y \mid y \delta)-(a \mid x)(x \mid y b \delta)+(a \mid y b \delta)(x \mid x)+(b \mid y)(y \mid x b \delta)-(b \mid x b \delta)(y \mid y)=0 \\
& -2(b \mid x y)(y \mid x b)+2(b \mid x b)(y \mid x y)+(a \mid x y b)(x \mid x)-(a \mid x)(x \mid x y b)-(b \mid x x y)(y \mid b)+(b \mid b)(y \mid x x y)=0 \\
& -2(b \mid x y)(y \mid a b)+2(b \mid a b)(y \mid x y)+(a \mid a y b)(x \mid x)-(a \mid x)(x \mid a y b)-(b \mid x a y)(y \mid b)+(b \mid b)(y \mid x a y)=0 \\
& 2(b \mid b \delta)(y \mid x y)-2(b \mid x y)(y \mid b \delta)+(a \mid y b \delta)(x \mid x)-(a \mid x)(x \mid y b \delta)-(b \mid x y \delta)(y \mid b)+(b \mid b)(y \mid x y \delta)=0 \\
& -(a \mid x)(x \mid x b b)+(a \mid x b b)(x \mid x)-(b \mid x x b)(y \mid b)+(b \mid b)(y \mid x x b)=0 \\
& -2(b \mid x b)(y \mid a b)+2(b \mid a b)(y \mid x b)-(a \mid x)(x \mid a b b)+(a \mid a b b)(x \mid x)-(b \mid x a b)(y \mid b)+(b \mid b)(y \mid x a b)=0 \\
& 2(b \mid b \delta)(y \mid x b)-2(b \mid x b)(y \mid b \delta)-(a \mid x)(x \mid b b \delta)+(a \mid b b \delta)(x \mid x)-(b \mid x b \delta)(y \mid b)+(b \mid b)(y \mid x b \delta)=0 \\
& 2(b \mid x b)(y \mid a y)-2(b \mid a y)(y \mid x b)+(a \mid x y b)(x \mid a)-(a \mid a)(x \mid x y b)+(b \mid b)(y \mid x a y)-(b \mid x a y)(y \mid b)=0 \\
& -2(b \mid a y)(y \mid a b)+2(b \mid a b)(y \mid a y)+(a \mid a y b)(x \mid a)-(a \mid a)(x \mid a y b)+(b \mid b)(y \mid a a y)-(b \mid a a y)(y \mid b)=0 \\
& 2(b \mid b \delta)(y \mid a y)-2(b \mid a y)(y \mid b \delta)+(a \mid y b \delta)(x \mid a)-(a \mid a)(x \mid y b \delta)+(b \mid b)(y \mid a y \delta)-(b \mid a y \delta)(y \mid b)=0
\end{aligned}
$$




$$
\begin{array}{r}
2(b \mid x b)(y \mid a b)-2(b \mid a b)(y \mid x b)+(a \mid x b b)(x \mid a)-(a \mid a)(x \mid x b b)+(b \mid b)(y \mid x a b)-(b \mid x a b)(y \mid b)=0 \\
(a \mid a b b)(x \mid a)-(a \mid a)(x \mid a b b)+(b \mid b)(y \mid a a b)-(b \mid a a b)(y \mid b)=0 \\
2(b \mid b \delta)(y \mid a b)-2(b \mid a b)(y \mid b \delta)+(a \mid b b \delta)(x \mid a)-(a \mid a)(x \mid b b \delta)+(b \mid b)(y \mid a b \delta)-(b \mid a b \delta)(y \mid b)=0 \\
-2(b \mid x y)(y \mid x b)+2(b \mid x b)(y \mid x y)-(b \mid y)(y \mid x x b)+(b \mid x x b)(y \mid y)-(b \mid x x y)(y \mid b)+(b \mid b)(y \mid x x y)=0 \\
-2(b \mid x y)(y \mid a b)+2(b \mid a b)(y \mid x y)-(b \mid y)(y \mid x a b)+(b \mid x a b)(y \mid y)-(b \mid x a y)(y \mid b)+(b \mid b)(y \mid x a y)=0 \\
2(b \mid b \delta)(y \mid x y)-2(b \mid x y)(y \mid b \delta)-(b \mid y)(y \mid x b \delta)+(b \mid x b \delta)(y \mid y)-(b \mid x y \delta)(y \mid b)+(b \mid b)(y \mid x y \delta)=0 \\
-2(b \mid a y)(y \mid x b)+2(b \mid x b)(y \mid a y)-(b \mid y)(y \mid x a b)+(b \mid x a b)(y \mid y)-(b \mid x a y)(y \mid b)+(b \mid b)(y \mid x a y)=0 \\
-2(b \mid a y)(y \mid a b)+2(b \mid a b)(y \mid a y)-(b \mid y)(y \mid a a b)+(b \mid a a b)(y \mid y)-(b \mid a a y)(y \mid b)+(b \mid b)(y \mid a a y)=0 \\
2(b \mid b \delta)(y \mid a y)-2(b \mid a y)(y \mid b \delta)-(b \mid y)(y \mid a b \delta)+(b \mid a b \delta)(y \mid y)-(b \mid a y \delta)(y \mid b)+(b \mid b)(y \mid a y \delta)=0 \\
-2(b \mid y \delta)(y \mid x b)+2(b \mid x b)(y \mid y \delta)+(b \mid x b \delta)(y \mid y)-(b \mid y)(y \mid x b \delta)-(b \mid x y \delta)(y \mid b)+(b \mid b)(y \mid x y \delta)=0 \\
-2(b \mid y \delta)(y \mid a b)+2(b \mid a b)(y \mid y \delta)+(b \mid a b \delta)(y \mid y)-(b \mid y)(y \mid a b \delta)-(b \mid a y \delta)(y \mid b)+(b \mid b)(y \mid a y \delta)=0 \\
2(b \mid b \delta)(y \mid y \delta)-2(b \mid y \delta)(y \mid b \delta)+(b \mid b \delta \delta)(y \mid y)-(b \mid y)(y \mid b \delta \delta)-(b \mid y \delta \delta)(y \mid b)+(b \mid b)(y \mid y \delta \delta)=0 \\
-2(a \mid y y)(x \mid y b)+2(a \mid y b)(x \mid y y)+(b \mid y y b)(y \mid y)-(b \mid y)(y \mid y y b)-(b \mid y y y)(y \mid b)+(b \mid b)(y \mid y y y)=0 \\
-2(a \mid y y)(x \mid b b)+2(a \mid b b)(x \mid y y)+(b \mid y b b)(y \mid y)-(b \mid y)(y \mid y b b)-(b \mid y y b)(y \mid b)+(b \mid b)(y \mid y y b)=0 \\
-(b \mid y)(y \mid y b b)+(b \mid y b b)(y \mid y)-(b \mid y y b)(y \mid b)+(b \mid b)(y \mid y y b)=0 \\
-2(a \mid y b)(x \mid b b)+2(a \mid b b)(x \mid y b)-(b \mid y)(y \mid b b b)+(b \mid b b b)(y \mid y)-(b \mid y b b)(y \mid b)+(b \mid b)(y \mid y b b)=0
\end{array}
$$

$$
\begin{aligned}
& (l \mid x x x)=-2(a \mid x \delta)(x \mid x x)+2(a \mid x x)(x \mid x \delta)-(a \mid x x \delta)(x \mid x)+(a \mid x)(x \mid x x \delta)-(a \mid \delta)(x \mid x x x)+(a \mid x x x)(x \mid \delta) \\
& (l \mid x x a)=2(a \mid x x)(x \mid a \delta)-2(a \mid a \delta)(x \mid x x)-(a \mid x a \delta)(x \mid x)+(a \mid x)(x \mid x a \delta)-(a \mid \delta)(x \mid x x a)+(a \mid x x a)(x \mid \delta) \\
& (l \mid x x \delta)=-2(a \mid \delta \delta)(x \mid x x)+2(a \mid x x)(x \mid \delta \delta)-(a \mid x \delta \delta)(x \mid x)+(a \mid x)(x \mid x \delta \delta)-(a \mid \delta)(x \mid x x \delta)+(a \mid x x \delta)(x \mid \delta) \\
& (l \mid x x a)=-2(a \mid x \delta)(x \mid x a)+2(a \mid x a)(x \mid x \delta)+(a \mid x)(x \mid x a \delta)-(a \mid x a \delta)(x \mid x)-(a \mid \delta)(x \mid x x a)+(a \mid x x a)(x \mid \delta) \\
& (l \mid x a a)=2(a \mid x a)(x \mid a \delta)-2(a \mid a \delta)(x \mid x a)+(a \mid x)(x \mid a a \delta)-(a \mid a a \delta)(x \mid x)-(a \mid \delta)(x \mid x a a)+(a \mid x a a)(x \mid \delta) \\
& (l \mid x a \delta)=-2(a \mid \delta \delta)(x \mid x a)+2(a \mid x a)(x \mid \delta \delta)+(a \mid x)(x \mid a \delta \delta)-(a \mid a \delta \delta)(x \mid x)-(a \mid \delta)(x \mid x a \delta)+(a \mid x a \delta)(x \mid \delta) \\
& (l \mid x x \delta)=-(a \mid x \delta \delta)(x \mid x)+(a \mid x)(x \mid x \delta \delta)-(a \mid \delta)(x \mid x x \delta)+(a \mid x x \delta)(x \mid \delta) \\
& (l \mid x a \delta)=2(a \mid x \delta)(x \mid a \delta)-2(a \mid a \delta)(x \mid x \delta)-(a \mid a \delta \delta)(x \mid x)+(a \mid x)(x \mid a \delta \delta)-(a \mid \delta)(x \mid x a \delta)+(a \mid x a \delta)(x \mid \delta) \\
& (l \mid x \delta \delta)=-2(a \mid \delta \delta)(x \mid x \delta)+2(a \mid x \delta)(x \mid \delta \delta)-(a \mid \delta \delta \delta)(x \mid x)+(a \mid x)(x \mid \delta \delta \delta)-(a \mid \delta)(x \mid x \delta \delta)+(a \mid x \delta \delta)(x \mid \delta) \\
& (l \mid x x a)=-2(a \mid x \delta)(x \mid x a)+2(a \mid x a)(x \mid x \delta)-(a \mid x x \delta)(x \mid a)+(a \mid a)(x \mid x x \delta)-(a \mid \delta)(x \mid x x a)+(a \mid x x a)(x \mid \delta)
\end{aligned}
$$


$(l \mid x a a)=-2(a \mid a \delta)(x \mid x a)+2(a \mid x a)(x \mid a \delta)-(a \mid x a \delta)(x \mid a)+(a \mid a)(x \mid x a \delta)-(a \mid \delta)(x \mid x a a)+(a \mid x a a)(x \mid \delta)$ $(l \mid x a \delta)=-2(a \mid \delta \delta)(x \mid x a)+2(a \mid x a)(x \mid \delta \delta)-(a \mid x \delta \delta)(x \mid a)+(a \mid a)(x \mid x \delta \delta)-(a \mid \delta)(x \mid x a \delta)+(a \mid x a \delta)(x \mid \delta)$ $(l \mid x a a)=-2(a \mid x \delta)(x \mid a a)+2(a \mid a a)(x \mid x \delta)-(a \mid x a \delta)(x \mid a)+(a \mid a)(x \mid x a \delta)-(a \mid \delta)(x \mid x a a)+(a \mid x a a)(x \mid \delta)$ $(l \mid a a a)=-2(a \mid a \delta)(x \mid a a)+2(a \mid a a)(x \mid a \delta)-(a \mid a a \delta)(x \mid a)+(a \mid a)(x \mid a a \delta)-(a \mid \delta)(x \mid a a a)+(a \mid a a a)(x \mid \delta)$ $(l \mid a a \delta)=-2(a \mid \delta \delta)(x \mid a a)+2(a \mid a a)(x \mid \delta \delta)-(a \mid a \delta \delta)(x \mid a)+(a \mid a)(x \mid a \delta \delta)-(a \mid \delta)(x \mid a a \delta)+(a \mid a a \delta)(x \mid \delta)$ $(l \mid x a \delta)=-2(a \mid x \delta)(x \mid a \delta)+2(a \mid a \delta)(x \mid x \delta)-(a \mid x \delta \delta)(x \mid a)+(a \mid a)(x \mid x \delta \delta)-(a \mid \delta)(x \mid a x \delta)+(a \mid a x \delta)(x \mid \delta)$ $(l \mid a a \delta)=-(a \mid a \delta \delta)(x \mid a)+(a \mid a)(x \mid a \delta \delta)-(a \mid \delta)(x \mid a a \delta)+(a \mid a a \delta)(x \mid \delta)$ $(l \mid a \delta \delta)=-2(a \mid \delta \delta)(x \mid a \delta)+2(a \mid a \delta)(x \mid \delta \delta)-(a \mid \delta \delta \delta)(x \mid a)+(a \mid a)(x \mid \delta \delta \delta)-(a \mid \delta)(x \mid a \delta \delta)+(a \mid a \delta \delta)(x \mid \delta)$ $(l \mid x y y)=-2(b \mid y \delta)(y \mid x y)+2(b \mid x y)(y \mid y \delta)-(a \mid y y \delta)(x \mid x)+(a \mid x)(x \mid y y \delta)-(a \mid \delta)(x \mid x y y)+(a \mid x y y)(x \mid \delta)$ $(l \mid x y b)=2(b \mid x y)(y \mid b \delta)-2(b \mid b \delta)(y \mid x y)-(a \mid y b \delta)(x \mid x)+(a \mid x)(x \mid y b \delta)-(a \mid \delta)(x \mid x y b)+(a \mid x y b)(x \mid \delta)$ $(l \mid x y b)=-2(b \mid y \delta)(y \mid x b)+2(b \mid x b)(y \mid y \delta)+(a \mid x)(x \mid y b \delta)-(a \mid y b \delta)(x \mid x)-(a \mid \delta)(x \mid x y b)+(a \mid x y b)(x \mid \delta)$ $(l \mid x b b)=2(b \mid x b)(y \mid b \delta)-2(b \mid b \delta)(y \mid x b)+(a \mid x)(x \mid b b \delta)-(a \mid b b \delta)(x \mid x)-(a \mid \delta)(x \mid x b b)+(a \mid x b b)(x \mid \delta)$ $(l \mid a y y)=-2(b \mid y \delta)(y \mid a y)+2(b \mid a y)(y \mid y \delta)-(a \mid y y \delta)(x \mid a)+(a \mid a)(x \mid y y \delta)-(a \mid \delta)(x \mid a y y)+(a \mid a y y)(x \mid \delta)$ $(l \mid a y b)=-2(b \mid b \delta)(y \mid a y)+2(b \mid a y)(y \mid b \delta)-(a \mid y b \delta)(x \mid a)+(a \mid a)(x \mid y b \delta)-(a \mid \delta)(x \mid a y b)+(a \mid a y b)(x \mid \delta)$ $(l \mid a y b)=-2(b \mid y \delta)(y \mid a b)+2(b \mid a b)(y \mid y \delta)-(a \mid y b \delta)(x \mid a)+(a \mid a)(x \mid y b \delta)-(a \mid \delta)(x \mid a y b)+(a \mid a y b)(x \mid \delta)$ $(l \mid a b b)=-2(b \mid b \delta)(y \mid a b)+2(b \mid a b)(y \mid b \delta)-(a \mid b b \delta)(x \mid a)+(a \mid a)(x \mid b b \delta)-(a \mid \delta)(x \mid a b b)+(a \mid a b b)(x \mid \delta)$ $(l \mid x y y)=-2(b \mid y \delta)(y \mid x y)+2(b \mid x y)(y \mid y \delta)+(b \mid y)(y \mid x y \delta)-(b \mid x y \delta)(y \mid y)-(a \mid \delta)(x \mid x y y)+(a \mid x y y)(x \mid \delta)$ $(l \mid x y b)=2(b \mid x y)(y \mid b \delta)-2(b \mid b \delta)(y \mid x y)+(b \mid y)(y \mid x b \delta)-(b \mid x b \delta)(y \mid y)-(a \mid \delta)(x \mid x y b)+(a \mid x y b)(x \mid \delta)$ $(l \mid a y y)=-2(b \mid y \delta)(y \mid a y)+2(b \mid a y)(y \mid y \delta)+(b \mid y)(y \mid a y \delta)-(b \mid a y \delta)(y \mid y)-(a \mid \delta)(x \mid a y y)+(a \mid a y y)(x \mid \delta)$ $(l \mid a y b)=2(b \mid a y)(y \mid b \delta)-2(b \mid b \delta)(y \mid a y)+(b \mid y)(y \mid a b \delta)-(b \mid a b \delta)(y \mid y)-(a \mid \delta)(x \mid a y b)+(a \mid a y b)(x \mid \delta)$ $(l \mid y y \delta)=-(b \mid y \delta \delta)(y \mid y)+(b \mid y)(y \mid y \delta \delta)-(a \mid \delta)(x \mid y y \delta)+(a \mid y y \delta)(x \mid \delta)$ $(l \mid y b \delta)=2(b \mid y \delta)(y \mid b \delta)-2(b \mid b \delta)(y \mid y \delta)-(b \mid b \delta \delta)(y \mid y)+(b \mid y)(y \mid b \delta \delta)-(a \mid \delta)(x \mid y b \delta)+(a \mid y b \delta)(x \mid \delta)$ $(l \mid x y b)=-2(b \mid y \delta)(y \mid x b)+2(b \mid x b)(y \mid y \delta)-(b \mid x y \delta)(y \mid b)+(b \mid b)(y \mid x y \delta)-(a \mid \delta)(x \mid x y b)+(a \mid x y b)(x \mid \delta)$ $(l \mid x b b)=-2(b \mid b \delta)(y \mid x b)+2(b \mid x b)(y \mid b \delta)-(b \mid x b \delta)(y \mid b)+(b \mid b)(y \mid x b \delta)-(a \mid \delta)(x \mid x b b)+(a \mid x b b)(x \mid \delta)$ $(l \mid a y b)=-2(b \mid y \delta)(y \mid a b)+2(b \mid a b)(y \mid y \delta)+(b \mid b)(y \mid a y \delta)-(b \mid a y \delta)(y \mid b)-(a \mid \delta)(x \mid a y b)+(a \mid a y b)(x \mid \delta)$ 


$$
\begin{aligned}
& (l \mid a b b)=-2(b \mid b \delta)(y \mid a b)+2(b \mid a b)(y \mid b \delta)+(b \mid b)(y \mid a b \delta)-(b \mid a b \delta)(y \mid b)-(a \mid \delta)(x \mid a b b)+(a \mid a b b)(x \mid \delta) \\
& (l \mid y b \delta)=-2(b \mid y \delta)(y \mid b \delta)+2(b \mid b \delta)(y \mid y \delta)-(b \mid y \delta \delta)(y \mid b)+(b \mid b)(y \mid y \delta \delta)-(a \mid \delta)(x \mid y b \delta)+(a \mid y b \delta)(x \mid \delta) \\
& (l \mid b b \delta)=-(b \mid b \delta \delta)(y \mid b)+(b \mid b)(y \mid b \delta \delta)-(a \mid \delta)(x \mid b b \delta)+(a \mid b b \delta)(x \mid \delta) \\
& (l \mid x y y)=2(a \mid y y)(x \mid x \delta)-2(a \mid x \delta)(x \mid y y)-(b \mid x y \delta)(y \mid y)+(b \mid y)(y \mid x y \delta)-(a \mid \delta)(x \mid x y y)+(a \mid x y y)(x \mid \delta) \\
& (l \mid a y y)=2(a \mid y y)(x \mid a \delta)-2(a \mid a \delta)(x \mid y y)-(b \mid a y \delta)(y \mid y)+(b \mid y)(y \mid a y \delta)-(a \mid \delta)(x \mid a y y)+(a \mid a y y)(x \mid \delta) \\
& (l \mid y y \delta)=-2(a \mid \delta \delta)(x \mid y y)+2(a \mid y y)(x \mid \delta \delta)-(b \mid y \delta \delta)(y \mid y)+(b \mid y)(y \mid y \delta \delta)-(a \mid \delta)(x \mid y y \delta)+(a \mid y y \delta)(x \mid \delta) \\
& (l \mid x y b)=2(a \mid y b)(x \mid x \delta)-2(a \mid x \delta)(x \mid y b)+(b \mid y)(y \mid x b \delta)-(b \mid x b \delta)(y \mid y)-(a \mid \delta)(x \mid x y b)+(a \mid x y b)(x \mid \delta) \\
& (l \mid a y b)=2(a \mid y b)(x \mid a \delta)-2(a \mid a \delta)(x \mid y b)+(b \mid y)(y \mid a b \delta)-(b \mid a b \delta)(y \mid y)-(a \mid \delta)(x \mid a y b)+(a \mid a y b)(x \mid \delta) \\
& (l \mid y b \delta)=-2(a \mid \delta \delta)(x \mid y b)+2(a \mid y b)(x \mid \delta \delta)+(b \mid y)(y \mid b \delta \delta)-(b \mid b \delta \delta)(y \mid y)-(a \mid \delta)(x \mid y b \delta)+(a \mid y b \delta)(x \mid \delta) \\
& (l \mid x y b)=-2(a \mid x \delta)(x \mid y b)+2(a \mid y b)(x \mid x \delta)-(b \mid x y \delta)(y \mid b)+(b \mid b)(y \mid x y \delta)-(a \mid \delta)(x \mid x y b)+(a \mid x y b)(x \mid \delta) \\
& (l \mid a y b)=2(a \mid y b)(x \mid a \delta)-2(a \mid a \delta)(x \mid y b)-(b \mid a y \delta)(y \mid b)+(b \mid b)(y \mid a y \delta)-(a \mid \delta)(x \mid a y b)+(a \mid a y b)(x \mid \delta) \\
& (l \mid y b \delta)=-2(a \mid \delta \delta)(x \mid y b)+2(a \mid y b)(x \mid \delta \delta)-(b \mid y \delta \delta)(y \mid b)+(b \mid b)(y \mid y \delta \delta)-(a \mid \delta)(x \mid y b \delta)+(a \mid y b \delta)(x \mid \delta) \\
& (l \mid x b b)=-2(a \mid x \delta)(x \mid b b)+2(a \mid b b)(x \mid x \delta)-(b \mid x b \delta)(y \mid b)+(b \mid b)(y \mid x b \delta)-(a \mid \delta)(x \mid x b b)+(a \mid x b b)(x \mid \delta) \\
& (l \mid a b b)=2(a \mid b b)(x \mid a \delta)-2(a \mid a \delta)(x \mid b b)-(b \mid a b \delta)(y \mid b)+(b \mid b)(y \mid a b \delta)-(a \mid \delta)(x \mid a b b)+(a \mid a b b)(x \mid \delta) \\
& (l \mid b b \delta)=-2(a \mid \delta \delta)(x \mid b b)+2(a \mid b b)(x \mid \delta \delta)-(b \mid b \delta \delta)(y \mid b)-(b \mid b)(y \mid b \delta \delta)-(a \mid \delta)(x \mid b b \delta)+(a \mid b b \delta)(x \mid \delta)
\end{aligned}
$$

\section{APPENDIX B: RELATIONS DUE TO MIRROR SYMMETRY}

The first order relations are the following:

$$
\begin{aligned}
& (x \mid x)^{2}-(x \mid a)(a \mid x)=1 \\
& (a \mid a)^{2}-(x \mid a)(a \mid x)=1 \\
& (x \mid a)[(x \mid x)-(a \mid a)]=0 \\
& (a \mid x)[(x \mid x)-(a \mid a)]=0 \\
& (y \mid y)^{2}-(y \mid b)(b \mid y)=1 \\
& (b \mid b)^{2}-(y \mid b)(b \mid y)=1 \\
& (y \mid b)[(y \mid y)-(b \mid b)]=0 \\
& (b \mid y)[(y \mid y)-(b \mid b)]=0 .
\end{aligned}
$$

These, combined with symplecticity, give $(x \mid x)=(a \mid a)$ and $(y \mid y)=(b \mid b)$. In addition,

$$
\begin{aligned}
& (x \mid \delta)[(x \mid x)+1]-(a \mid \delta)(x \mid a)=0 \\
& (a \mid \delta)[1-(a \mid a)]+(x \mid \delta)(a \mid x)=0 .
\end{aligned}
$$

The second order relations are the following:

$$
\begin{gathered}
-(a \mid x x)(x \mid a)+(a \mid x)^{2}(x \mid a a)-(a \mid x)(x \mid x)(x \mid x a) \\
+(x \mid x)(x \mid x x)+(x \mid x)^{2}(x \mid x x)=0 \\
-(a \mid x a)(x \mid a)+2(a \mid a)(a \mid x)(x \mid a a)-(a \mid x)(x \mid a)(x \mid x a) \\
+(x \mid x)(x \mid x a)-(a \mid a)(x \mid x)(x \mid x a)+2(x \mid a)(x \mid x)(x \mid x x)=0 \\
-(a \mid x \delta)(x \mid a)+2(a \mid \delta)(a \mid x)(x \mid a a)-(a \mid x)(x \mid a \delta) \\
-(a \mid x)(x \mid \delta)(x \mid x a)-(a \mid \delta)(x \mid x)(x \mid x a)+2(x \mid x)(x \mid x \delta) \\
+2(x \mid \delta)(x \mid x)(x \mid x x)=0 \\
-(a \mid a a)(x \mid a)+(a \mid a)^{2}(x \mid a a)+(x \mid a a)(x \mid x) \\
-(a \mid a)(x \mid a)(x \mid x a)+(x \mid a)^{2}(x \mid x x)=0
\end{gathered}
$$




$$
\begin{array}{r}
-(a \mid a \delta)(x \mid a)+2(a \mid a)(a \mid \delta)(x \mid a a)-(a \mid a)(x \mid a \delta) \\
+(x \mid a \delta)(x \mid x)-(a \mid \delta)(x \mid a)(x \mid x a)-(a \mid a)(x \mid \delta)(x \mid x a) \\
+(x \mid a)(x \mid x \delta)+2(x \mid a)(x \mid \delta)(x \mid x x)=0 \\
-(a \mid y y)(x \mid a)+(b \mid y)^{2}(x \mid b b)+(x \mid x)(x \mid y y) \\
-(b \mid y)(x \mid y b)(y \mid y)+(x \mid y y)(y \mid y)^{2}=0 \\
-(a \mid y b)(x \mid a)+2(b \mid b)(b \mid y)(x \mid b b)+(x \mid x)(x \mid y b) \\
-(b \mid y)(x \mid y b)(y \mid b)-(b \mid b)(x \mid y b)(y \mid y) \\
+2(x \mid y y)(y \mid b)(y \mid y)=0 \\
-(a \mid b b)(x \mid a)+(b \mid b)^{2}(x \mid b b)+(x \mid b b)(x \mid x) \\
-(b \mid b)(x \mid y b)(y \mid b)+(x \mid y y)(y \mid b)^{2}=0 \\
-(a \mid \delta \delta)(x \mid a)+(a \mid \delta)^{2}(x \mid a a)-(a \mid \delta)(x \mid a \delta)+(x \mid \delta \delta) \\
+(x \mid \delta \delta)(x \mid x)-(a \mid \delta)(x \mid \delta)(x \mid x a)+(x \mid \delta)(x \mid x \delta) \\
+(x \mid \delta)^{2}(x \mid x x)=0
\end{array}
$$

$(a \mid a a)(a \mid x)^{2}-(a \mid a)(a \mid x x)-(a \mid x)(a \mid x a)(x \mid x)$

$$
+(a \mid x x)(x \mid x)^{2}+(a \mid x)(x \mid x x)=0
$$

$2(a \mid a)(a \mid a a)(a \mid x)-(a \mid a)(a \mid x a)-(a \mid x)(a \mid x a)(x \mid a)$

$-(a \mid a)(a \mid x a)(x \mid x)+2(a \mid x x)(x \mid a)(x \mid x)$

$$
+(a \mid x)(x \mid x a)=0
$$

$-(a \mid a \delta)(a \mid x)+2(a \mid a a)(a \mid \delta)(a \mid x)-(a \mid a)(a \mid x \delta)$

$-(a \mid x)(a \mid x a)(x \mid \delta)-(a \mid \delta)(a \mid x a)(x \mid x)+(a \mid x \delta)(x \mid x)$

$$
+2(a \mid x x)(x \mid \delta)(x \mid x)+(a \mid x)(x \mid x \delta)=0
$$

$-(a \mid a)(a \mid a a)+(a \mid a)^{2}(a \mid a a)-(a \mid a)(a \mid x a)(x \mid a)$

$$
+(a \mid x x)(x \mid a)^{2}+(a \mid x)(x \mid a a)=0
$$

$-2(a \mid a)(a \mid a \delta)+2(a \mid a)(a \mid a a)(a \mid \delta)-(a \mid \delta)(a \mid x a)(x \mid a)$

$+(a \mid x \delta)(x \mid a)+(a \mid x)(x \mid a \delta)-(a \mid a)(a \mid x a)(x \mid \delta)$

$$
+2(a \mid x x)(x \mid a)(x \mid \delta)=0
$$

$-(a \mid a)(a \mid y y)+(a \mid b b)(b \mid y)^{2}+(a \mid x)(x \mid y y)$

$$
-(a \mid y b)(b \mid y)(y \mid y)+(a \mid y y)(y \mid y)^{2}=0
$$

$-(a \mid a)(a \mid y b)+2(a \mid b b)(b \mid b)(b \mid y)+(a \mid x)(x \mid y b)$

$-(a \mid y b)(b \mid y)(y \mid b)-(a \mid y b)(b \mid b)(y \mid y)$

$$
+2(a \mid y y)(y \mid b)(y \mid y)=0
$$

$$
\begin{aligned}
-(a \mid a)(a \mid b b)+(a \mid b b)(b \mid b)^{2}+(a \mid x)(x \mid b b) & \\
-(a \mid y b)(b \mid b)(y \mid b) & +(a \mid y y)(y \mid b)^{2}=0 \\
(a \mid a \delta)(a \mid \delta)+(a \mid a a)(a \mid \delta)^{2}+(a \mid \delta \delta)- & (a \mid a)(a \mid \delta \delta) \\
-(a \mid \delta)(a \mid x a)(x \mid \delta)+(a \mid x \delta)(x \mid \delta)+ & (a \mid x x)(x \mid \delta)^{2} \\
& +(a \mid x)(x \mid \delta \delta)=0
\end{aligned}
$$

$(a \mid x)(b \mid y)(y \mid a b)-(b \mid x y)(y \mid b)-(b \mid y)(x \mid x)(y \mid x b)$

$$
-(a \mid x)(y \mid a y)(y \mid y)+(y \mid x y)(y \mid y)+(x \mid x)(y \mid x y)(y \mid y)=0
$$

$(a \mid x)(b \mid b)(y \mid a b)-(b \mid x b)(y \mid b)-(a \mid x)(y \mid a y)(y \mid b)$

$-(b \mid b)(x \mid x)(y \mid x b)+(x \mid x)(y \mid b)(y \mid x y)+(y \mid x b)(y \mid y)=0$

$(a \mid a)(b \mid y)(y \mid a b)-(b \mid a y)(y \mid b)-(b \mid y)(x \mid a)(y \mid x b)$

$+(y \mid a y)(y \mid y)-(a \mid a)(y \mid a y)(y \mid y)+(x \mid a)(y \mid x y)(y \mid y)=0$

$(a \mid a)(b \mid b)(y \mid a b)-(b \mid a b)(y \mid b)-(a \mid a)(y \mid a y)(y \mid b)$

$-(b \mid b)(x \mid a)(y \mid x b)+(x \mid a)(y \mid b)(y \mid x y)+(y \mid a b)(y y)=0$

$(a \mid \delta)(b \mid y)(y \mid a b)-(b \mid y \delta)(y \mid b)-(b \mid y)(y \mid b \delta)$

$-(b \mid y)(x \mid \delta)(y \mid x b)-(a \mid \delta)(y \mid a y)(y \mid y)$

$$
+(x \mid \delta)(y \mid x y)(y \mid y)+2(y \mid y)(y \mid y \delta)=0
$$

$(a \mid \delta)(b \mid b)(y \mid a b)-(b \mid b \delta)(y \mid b)-(a \mid \delta)(y \mid a y)(y \mid b)$

$-(b \mid b)(y \mid b \delta)-(b \mid b)(x \mid \delta)(y \mid x b)+(x \mid \delta)(y \mid b)(y \mid x y)$

$$
+(y \mid b \delta)(y \mid y)+(y \mid b)(y \mid y \delta)=0
$$

$$
\begin{aligned}
- & (b \mid b)(b \mid x y)+(a \mid x)(b \mid a b)(b \mid y)-(b \mid x b)(b \mid y)(x \mid x) \\
& +(b \mid y)(y \mid x y)-(a \mid x)(b \mid a y)(y \mid y)+(b \mid x y)(x \mid x)(y \mid y)=0
\end{aligned}
$$

$(a \mid x)(b \mid a b)(b \mid b)-(b \mid b)(b \mid x b)-(b \mid b)(b \mid x b)(x \mid x)$

$-(a \mid x)(b \mid a y)(y \mid b)+(b \mid x y)(x \mid x)(y \mid b)+(b \mid y)(y \mid x b)=0$

$-(b \mid a y)(b \mid b)+(a \mid a)(b \mid a b)(b \mid y)-(b \mid x b)(b \mid y)(x \mid a)$

$+(b \mid y)(y \mid a y)-(a \mid a)(b \mid a y)(y \mid y)+(b \mid x y)(x \mid a)(y \mid y)=0$

$-(b \mid a b)(b \mid b)+(a \mid a)(b \mid a b)(b \mid b)-(b \mid b)(b \mid x b)(x \mid a)$

$+(b \mid y)(y \mid a b)-(a \mid a)(b \mid a y)(y \mid b)+(b \mid x y)(x \mid a)(y \mid b)=0$

$(a \mid \delta)(b \mid a b)(b \mid y)-(b \mid b \delta)(b \mid y)-(b \mid b)(b \mid y \delta)$

$-(b \mid x b)(b \mid y)(x \mid \delta)-(a \mid \delta)(b \mid a y)(y \mid y)+(b \mid y \delta)(y \mid y)$

$+(b \mid x y)(x \mid \delta)(y \mid y)+(b \mid y)(y \mid y \delta)=0$ 


$$
\begin{aligned}
&(a \mid \delta)(b \mid a b)(b \mid b)-2(b \mid b)(b \mid b \delta)-(b \mid b)(b \mid x b)(x \mid \delta) \\
&-(a \mid \delta)(b \mid a y)(y \mid b)+(b \mid y \delta)(y \mid b)+(b \mid x y)(x \mid \delta)(y \mid b) \\
&+(b \mid y)(y \mid b \delta)=0 .
\end{aligned}
$$

[1] Y. Yano, in Proceedings of the 17th International Conference on Cyclotrons and their Applications, Tokyo, Japan, 2004, pp. 169-173.

[2] W. Henning, Nucl. Phys. A734, 654 (2004).

[3] M. Lewitowicz, Nucl. Phys. A734, 645 (2004).

[4] J. Nolen, Nucl. Phys. A734, 661 (2004).

[5] B. M. Sherrill, Nucl. Instrum. Methods Phys. Res., Sect. B 204, 765 (2003).

[6] Opportunities in Nuclear Science, edited by J. Symons, Technical report, NSAC, 2002.

[7] RISAC, Scientific Opportunities with a Rare-Isotope Facility in the United States, Technical report, NRC, 2006.

[8] J. Alonso, A. Chatterjee, and C. A. Tobias, IEEE Trans. Nucl. Sci. 26, 3003 (1979).

[9] K.-H. Schmidt, E. Hanelt, H. Geissel, G. Munzenberg, and J. P. Dufour, Nucl. Instrum. Methods Phys. Res., Sect. A 260, 287 (1987).

[10] J. P. Dufour and R. Del Moral, Nucl. Instrum. Methods Phys. Res., Sect. A 248, 267 (1986).

[11] H. Geissel et al., Nucl. Instrum. Methods Phys. Res., Sect. B 70, 286 (1992).

[12] T. Kubo, Nucl. Instrum. Methods Phys. Res., Sect. B 204, 97 (2003).

[13] G. Savard, J. Schwartz, J. Caggiano, J. P. Greene, A. Heinz, M. Maier, D. Seweryniak, and B. J. Zabransky,
Nucl. Phys. A701, 292 (2002).

[14] B. M. Sherrill, D. J. Morrissey, J. A. Nolen, and J. A. Winger, Nucl. Instrum. Methods Phys. Res., Sect. B 56, 1106 (1991).

[15] D. J. Morrissey, B. M. Sherrill, M. Steiner, and I. Wiedenhoever, Nucl. Instrum. Methods Phys. Res., Sect. B 204, 90 (2003).

[16] H. Geissel et al., Nucl. Instrum. Methods Phys. Res., Sect. B 204, 71 (2003).

[17] M. Berz, Modern Map Methods in Particle Beam Physics (Academic Press, San Diego, 1999).

[18] H. Wollnik and M. Berz, Nucl. Instrum. Methods 238, 127 (1985).

[19] S. Wolfram, The MATHEMATICA Book (Wolfram Media, Champaign, IL, 2003), 5th ed.

[20] J.D. Jackson, Classical Electrodynamics (Wiley, New York, 1975).

[21] K. Makino and M. Berz, Nucl. Instrum. Methods Phys. Res., Sect. A 558, 346 (2006).

[22] W. Wan and M. Berz, Phys. Rev. E 54, 2870 (1996).

[23] J. Maloney, Master's thesis, Northern Illinois University, DeKalb, IL, 2006.

[24] Shashikant Manikonda, Jerry Nolen, Martin Berz, Kyoko Makino, and Gabi Weizman, Technical Report MSUHEP060412, Michigan State University, 2006.

[25] M. R. James, G. W. McKinney, J.S. Hendricks, and M. Moyers, Nucl. Instrum. Methods Phys. Res., Sect. A 562, 819 (2006).

[26] L. Bandura, M. Berz, B. Erdelyi, K. Makino, J. Maloney, S. Manikonda, and J.A. Nolen, in Proceedings of the International Nuclear Physics Conference, Tokyo, Japan, 2007. 